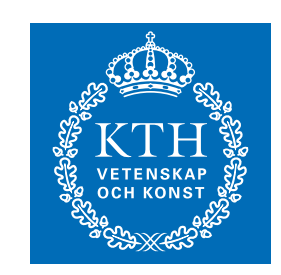

КTH Electrical Engineering

\title{
Cooperative Multicell Precoding: Rate Region Characterization and Distributed Strategies with Instantaneous and Statistical CSI
}

\author{
IEEE TRANSACTIONS ON SIGNAL PROCESSING \\ Volume 58, Issue 8, Pages 4298-4310, August 2010.
}

Copyright (c) 2010 IEEE. Reprinted from Trans. on Signal Processing.

This material is posted here with permission of the IEEE. Such permission of the IEEE does not in any way imply IEEE endorsement of any of the KTH Royal Institute of Technology's products or services. Internal or personal use of this material is permitted. However, permission to reprint/republish this material for advertising or promotional purposes or for creating new collective works for resale or redistribution must be obtained from the IEEE by writing to pubs-permissions@ieee.org.

By choosing to view this document, you agree to all provisions of the copyright laws protecting it.

\author{
EMIL BJÖRNSON, RANDA ZAKHOUR, \\ DAVID GESBERT, AND BJÖRN OTTERSTEN
}

Stockholm 2010

\author{
KTH Royal Institute of Technology \\ ACCESS Linnaeus Center \\ Signal Processing Lab
}

DOI: $10.1109 /$ TSP.2010.2049996

KTH Report: IR-EE-SB 2010:005 


\title{
Cooperative Multicell Precoding: Rate Region Characterization and Distributed Strategies With Instantaneous and Statistical CSI
}

\author{
Emil Björnson, Student Member, IEEE, Randa Zakhour, Student Member, IEEE, \\ David Gesbert, Senior Member, IEEE, and Björn Ottersten, Fellow, IEEE
}

\begin{abstract}
Base station cooperation is an attractive way of increasing the spectral efficiency in multiantenna communication. By serving each terminal through several base stations in a given area, intercell interference can be coordinated and higher performance achieved, especially for terminals at cell edges. Most previous work in the area has assumed that base stations have common knowledge of both data dedicated to all terminals and full or partial channel state information (CSI) of all links. Herein, we analyze the case of distributed cooperation where each base station has only local CSI, either instantaneous or statistical. In the case of instantaneous CSI, the beamforming vectors that can attain the outer boundary of the achievable rate region are characterized for an arbitrary number of multiantenna transmitters and single-antenna receivers. This characterization only requires local CSI and justifies distributed precoding design based on a novel virtual signal-to-interference noise ratio (SINR) framework, which can handle an arbitrary SNR and achieves the optimal multiplexing gain. The local power allocation between terminals is solved heuristically. Conceptually, analogous results for the achievable rate region characterization and precoding design are derived in the case of local statistical CSI. The benefits of distributed cooperative transmission are illustrated numerically, and it is shown that most of the performance with centralized cooperation can be obtained using only local CSI.
\end{abstract}

Index Terms-Coordinated multipoint (CoMP), network multiple-input-multiple-output (MIMO), base station cooperation, distributed precoding, rate region, virtual signal-to-interference noise ratio (SINR).

Manuscript received September 26, 2009; revised January 11, 2010, March 26, 2010; accepted April 27, 2010. Date of publication May 10, 2010; date of current version July 14, 2010. The associate editor coordinating the review of this manuscript and approving it for publication was Prof. Huaiyu Dai. The research leading to these results has received funding from the European Research Council under the European Community's Seventh Framework Programme (FP7/2007-2013)/ERC Grant Agreement No. 228044. This work is supported in part by the FP6 project Cooperative and Opportunistic Communications in Wireless Networks (COOPCOM), Project Number: FP6-033533. This work was also supported in part by the French ANR-funded project ORMAC. This paper was previously presented in part at the IEEE Global Communications Conference (GLOBECOM), 2009.

E. Björnson and B. Ottersten are with the Signal Processing Laboratory, ACCESS Linnaeus Center, KTH Royal Institute of Technology, SE-100 44 Stockholm, Sweden (e-mail: emil.bjornson@ee.kth.se; bjorn.ottersten@ee.kth.se).

B. Ottersten is also with the University of Luxembourg, L-1359 LuxembourgKirchberg, Luxembourg.

R. Zakhour and D. Gesbert are with the Mobile Communications Department, EURECOM, 06560 Sophia Antipolis, France (e-mail: randa.zakhour@eurecom.fr; david.gesbert@eurecom.fr).

Color versions of one or more figures in this paper are available online at http://ieeexplore.ieee.org.

Digital Object Identifier 10.1109/TSP.2010.2049996

\section{INTRODUCTION}

$\mathbf{T}$ HE performance of cellular communication systems can be greatly improved by multiple-input-multiple-output (MIMO) techniques. Many algorithms have been proposed for the single-cell downlink scenario, where a base station communicates simultaneously with multiple terminals [1]. These approaches exploit various amounts of channel state information (CSI) and improve the throughput by optimizing the received signal gain and limiting the intracell interference. In multicell scenarios, these single-cell techniques are however obliged to treat the interference from adjacent cells as noise, resulting in a fundamental limitation on the performance [2]-[5] - especially for terminals close to cell edges.

In recent years, base station coordination (also known as network MIMO [4]) has been analyzed as a means of handling intercell interference. In principle, all base stations might share their CSI and data through backhaul links, which enable coordinated transmission that manages the interference as in a single cell with either total [6] or per-group-of-antennas power constraints [7]. Unfortunately, the demands on backhaul capacity and computational power scale rapidly with the number of cells [8], [9], which makes this approach unsuitable for practical systems. Thus, there is a great interest in distributed forms of cooperation that reduce the backhaul signaling and precoding complexity, while still benefiting from robust interference control [9]-[12]. Two major considerations in the design of such schemes are to which extent the cooperation is managed centrally (requires CSI sharing) and whether each terminal should be served by multiple base stations (requires data sharing).

We consider the scenario of base stations equipped with multiple antennas and terminals with a single antenna each. In this context, the multiple-input-single-output interference channel (MISO IC) represents the special case when each base station only serves a single unique terminal, but can share CSI to manage coterminal interference. Although each base station aims at maximizing the rate achieved by its own terminal, cooperation over the MISO IC can greatly improve the performance [13]. The achievable rate region was characterized in [14] and the authors proposed a game-theoretic precoding design based on full CSI sharing [13]. Distributed precoding that only exploits locally available CSI can be achieved when each base station balances the ratio between signal gain at the intended terminal and the interference caused at other terminals [15]-[17]. Recently, this approach has been shown to attain optimal rate points [17]. 
In this paper, we consider distributed network MIMO where the cooperating base stations share knowledge of the data symbols but have local CSI only, thereby reducing the feedback load on the uplink and avoiding cell-to-cell CSI exchange. The fundamental difference from the MISO IC is that multiple base stations can cooperate on serving each terminal, which means that the achievable rate region is larger [18]. In addition, the number of terminals is not limited by the number of base stations. In this paper, we derive a characterization of the optimal linear precoding strategy, which formally justifies a distributed approach that treats the system as a superposition of broadcast channels. This leads to novel distributed beamforming and power allocation strategies. The major contributions are the following.

- We characterize the achievable rate region for network MIMO with an arbitrary number of links and antennas at the transmitters, and either instantaneous or statistical CSI. The optimal beamformers belong to a certain subspace defined using local CSI. This parametrization provides understanding and a structure for heuristic precoding.

- We propose a distributed virtual SINR framework based on uplink-downlink duality theory [19]. This framework is used for distributed beamforming design and power allocation with local instantaneous CSI, and handles an arbitrary number of links. It achieves the optimal multiplexing gain and numerical examples show good and stable performance at all SNRs, which makes it more practical than distributed maximum ratio transmission (MRT) and zeroforcing (ZF).

- We extend this framework to handle beamforming design with local statistical CSI, for cases when instantaneous fading information is unavailable. A heuristic power allocation scheme is also proposed under these conditions.

Preliminary results with two base stations and two terminals were presented in [18]. The performance and complexity differences between centralized and distributed precoding are discussed in [20]. An alternative approach based on superposition of ICs is analyzed in [21].

Notations: Boldface (lower case) is used for column vectors, $\mathbf{X}$, and (upper case) for matrices, $\mathbf{X}$. Let $\mathbf{X}^{T}, \mathbf{X}^{H}$, and $\mathbf{X}^{*}$ denote the transpose, the conjugate transpose, and the conjugate of $\mathbf{X}$, respectively. The orthogonal projection matrix onto the column space of $\mathbf{X}$ is $\Pi_{\mathbf{X}}=\mathbf{X}\left(\mathbf{X}^{H} \mathbf{X}\right)^{-1} \mathbf{X}^{H}$, and that onto its orthogonal complement is $\Pi_{\mathbf{X}}^{\perp}=\mathbf{I}-\boldsymbol{\Pi}_{\mathbf{X}}$, where $\mathbf{I}$ is the identity matrix. $\mathcal{C N}(\overline{\mathbf{x}}, \mathbf{Q})$ is used to denote circularly symmetric complex Gaussian random vectors, where $\overline{\mathbf{x}}$ is the mean and $\mathbf{Q}$ is the covariance matrix.

\section{SySTEM MODEL}

The communication scenario herein consists of $K_{r}$ single-antenna receivers ${ }^{1}$ (e.g., active mobile terminals) and $K_{t}$ transmitters (e.g., base stations in a cellular system) equipped with $N_{t}$ antennas each. The $j$ th transmitter and $k$ th receiver are denoted $\mathrm{BS}_{j}$ and $\mathrm{MS}_{k}$, respectively, for $j \in\left\{1, \ldots, K_{t}\right\}$ and $k \in\left\{1, \ldots, K_{r}\right\}$. This setup is illustrated in Fig. 1 for $N_{t}=8$. Let $\mathbf{x}_{j} \in \mathbb{C}^{N_{t}}$ be the signal transmitted by $\mathrm{BS}_{j}$ and let the corresponding received signal at $\mathrm{MS}_{k}$ be denoted by $y_{k} \in \mathbb{C}$. The

\footnotetext{
${ }^{1}$ The results herein also apply to simple multi-antenna receivers that fix their
} receive beamforming (e.g., antenna selection) prior to base station optimization.

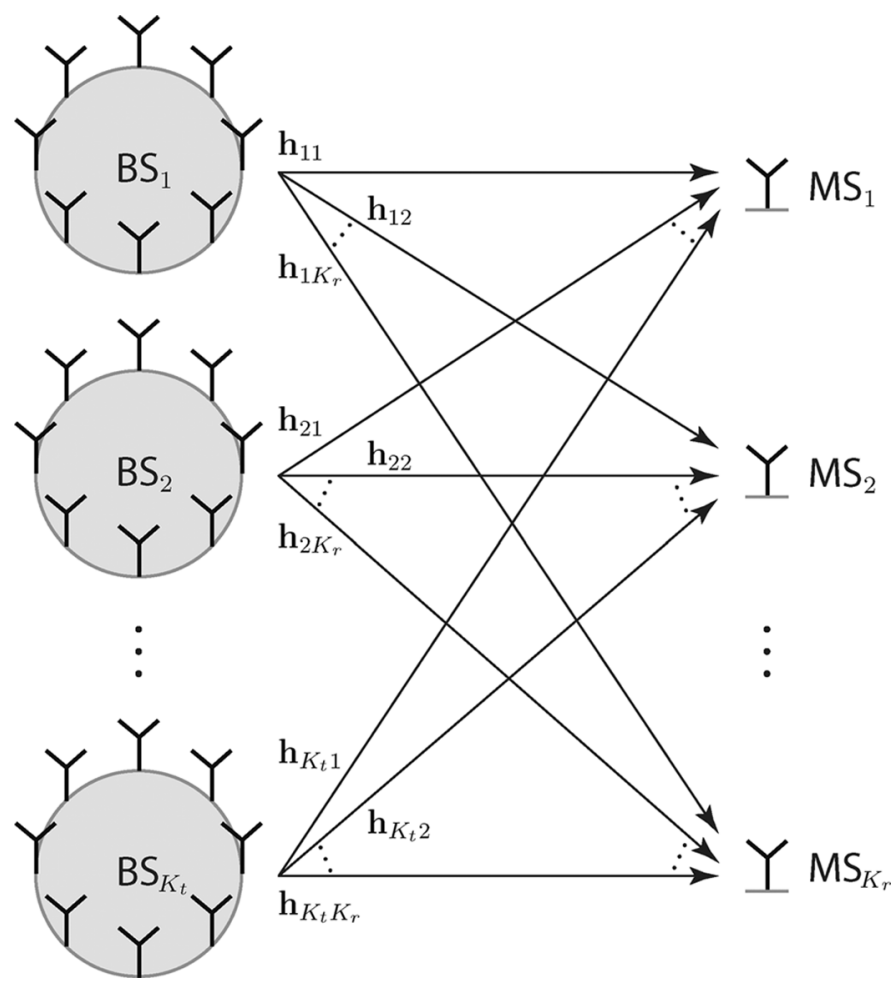

Fig. 1. The basic scenario with $K_{t}$ base stations and $K_{r}$ terminals (illustrated for $N_{t}=8$ transmit antennas).

propagation channel to $\mathrm{MS}_{k}$ is assumed to be narrowband, flat and Rayleigh fading with the system model

$$
y_{k}=\sum_{j=1}^{K_{t}} \mathbf{h}_{j k}^{H} \mathbf{x}_{j}+n_{k}
$$

where $\mathbf{h}_{j k} \in \mathcal{C N}\left(\mathbf{0}, \mathbf{Q}_{j k}\right)$ represents the channel between $\mathrm{BS}_{j}$ and $\mathrm{MS}_{k}$ and $n_{k} \in \mathcal{C} \mathcal{N}\left(0, \sigma_{k}^{2}\right)$ is white additive noise. The channel correlation matrix $\mathbf{Q}_{j k}=\mathbb{E}\left\{\mathbf{h}_{j k} \mathbf{h}_{j k}^{H}\right\} \in \mathbb{C}^{n_{T} \times n_{T}}$ is positive semi-definite. Throughout the paper, each receiver $\mathrm{MS}_{k}$ has full local CSI (i.e., perfect estimates of $\mathbf{h}_{j k}$ for $j=$ $\left.1, \ldots, K_{t}\right)$. At the transmitter side, we will distinguish between two different types of local CSI:

- Local Instantaneous CSI: $\mathrm{BS}_{j}$ knows the current channel vector $\mathbf{h}_{j k}$ and the noise power $\sigma_{k}^{2}$, for $k=1, \ldots, K_{r}$.

- Local Statistical CSI: $\mathrm{BS}_{j}$ knows the statistics of $\mathbf{h}_{j k}$ (e.g., type of distribution and $\mathbf{Q}_{j k}$ ) and the noise power $\sigma_{k}^{2}$, for $k=1, \ldots, K_{r}$.

Observe that in both cases, the philosophy is that transmitters only have CSI that can be obtained locally (either through feedback or reverse-link estimation [22]). Hence, there is no exchange of CSI between them, thus allowing the scalability of multicell cooperation to large and dense networks. For simplicity, each transmitter has CSI for its links to all receivers, which is nonscalable when the resources for CSI acquisition are limited. However, it is still a good model for large networks as most terminals will be far away from any given transmitter and thus have negligibly weak channel gains.

\section{A. Cooperative Multicell Precoding}

Let $s_{k} \in \mathcal{C N}(0,1)$ be the data symbol intended for $\mathrm{MS}_{k}$. Unlike the MISO IC [13]-[17], we assume that the data symbols 
intended for all receivers are available at all transmitters. This enables cooperative precoding techniques, where each receiver is served simultaneously by all the transmitters in the area ${ }^{2}$. Herein, we will consider distributed linear precoding where each transmitter selects its beamforming vectors independently using only local CSI, as defined above. Proper transmission synchronization is however required to avoid intersymbol interference. The signal transmitted by $\mathrm{BS}_{j}$ is

$$
\mathbf{x}_{j}=\sum_{k=1}^{K_{r}} \sqrt{p_{j k}} \mathbf{w}_{j k} s_{k}
$$

where the beamforming vectors $\mathbf{w}_{j k}$ have unit norms (i.e., $\left\|\mathbf{w}_{j k}\right\|=1$ ) and $p_{j k}$ represents the power allocated for transmission to $\mathrm{MS}_{k}$ from $\mathrm{BS}_{j} . \mathrm{BS}_{j}$ is subject to an individual average power constraint of $P_{j}$; that is, $\mathbb{E}\left\{\left\|\mathbf{x}_{j}\right\|^{2}\right\}=\sum_{k=1}^{K_{r}} p_{j k} \leq P_{j}$. Thus, the main differences between the scenario at hand and the MISO broadcast channel (BC) is that in the latter all antennas are controlled by a central unit with CSI of all links and a joint power constraint.

When the receivers treat coterminal interference as noise, the instantaneous SINR at $\mathrm{MS}_{k}$ is

$$
\operatorname{SINR}_{k}=\frac{\left|\sum_{j=1}^{K_{t}} \sqrt{p_{j k}} \mathbf{h}_{j k}^{H} \mathbf{w}_{j k}\right|^{2}}{\sum_{\substack{k=1 \\ k \neq k}}^{K_{r}}\left|\sum_{j=1}^{K_{t}} \sqrt{p_{j \bar{k}}} \mathbf{h}_{j k}^{H} \mathbf{w}_{j \bar{k}}\right|^{2}+\sigma_{k}^{2}}
$$

for $k=1, \ldots, K_{r}$. The maximal achievable instantaneous transmission rate is accordingly $R_{k}=\log _{2}\left(1+\mathrm{SINR}_{k}\right)$.

In the case of local statistical CSI at each transmitter, we introduce the notation $a_{\bar{k} k} \triangleq \sum_{j=1}^{K_{t}} \sqrt{p_{j \bar{k}}} \mathbf{h}_{j k}^{H} \mathbf{w}_{j \bar{k}}, \mathbf{S}_{k} \triangleq$ $\sum_{j=1}^{K_{t}} \mathbf{W}_{j}^{H} \mathbf{Q}_{j k} \mathbf{W}_{j}$, and $\mathbf{W}_{j} \triangleq\left[\sqrt{p_{j 1}} \mathbf{w}_{j 1} \cdots \sqrt{p_{j K_{r}}} \mathbf{w}_{j K_{r}}\right]$. The collective beamforming matrix $\mathbf{W}_{j}$ is statistically independent of the instantaneous channel realizations $\mathbf{h}_{j k}$, since it is only based on statistical CSI. Then, the stochastic behavior of the SINR in (3), seen by the transmitters, is clarified by the alternative expression

$$
\begin{aligned}
\operatorname{SINR}_{k} & =\frac{\left|a_{k k}\right|^{2}}{\sum_{\substack{k=1 \\
k \neq k}}^{K_{r}}\left|a_{\bar{k} k}\right|^{2}+\sigma_{k}^{2}} \\
\text { with } \mathbf{a}_{k} & =\left[a_{1 k} \ldots a_{K_{r} k}\right]^{H} \in \mathcal{C N}\left(\mathbf{0}, \mathbf{S}_{k}\right) .
\end{aligned}
$$

When the transmitters only have statistical CSI, they can only optimize an average performance measure. Herein, we therefore consider the expected achievable transmission rate, $\mathbb{E}\left\{R_{k}\right\}=$ $\mathbb{E}\left\{\log _{2}\left(1+\mathrm{SINR}_{k}\right)\right\}$. Using the notation introduced above, it can be calculated using the next theorem. The result will be used for precoding design in Section IV-B.

\footnotetext{
${ }^{2}$ This assumption will ease the exposition, but in practice the subset of terminals served by a given base station will be determined by a scheduler. This scheduling problem is however beyond the scope of this paper.
}

Theorem 1: Let $\tilde{\mathbf{S}}_{k}$ be the matrix obtained by removing the $k$ th column and $k$ th row of $\mathbf{S}_{k}$. Then

$$
\begin{aligned}
\mathbb{E}\left\{\log _{2}\left(1+\operatorname{SINR}_{k}\right)\right\} & \sum_{m=1}^{\operatorname{rank}\left(\mathbf{S}_{k}\right)} \frac{e^{\frac{\sigma_{k}^{2}}{\mu_{m}}} E_{1}\left(\frac{\sigma_{k}^{2}}{\mu_{m}}\right)}{\log (2) \prod_{l \neq m}\left(1-\frac{\mu_{l}}{\mu_{m}}\right)} \\
& -\sum_{m=1}^{\operatorname{rank}\left(\tilde{\mathbf{S}}_{k}\right)} \frac{e^{\frac{\sigma_{k}^{2}}{\lambda_{m}}} E_{1}\left(\frac{\sigma_{k}^{2}}{\lambda_{m}}\right)}{\log (2) \prod_{l \neq m}\left(1-\frac{\lambda_{l}}{\lambda_{m}}\right)}
\end{aligned}
$$

where $\mu_{1}, \ldots, \mu_{\operatorname{rank}\left(\mathbf{S}_{k}\right)}$ and $\lambda_{1}, \ldots, \lambda_{\operatorname{rank}\left(\tilde{\mathbf{S}}_{k}\right)}$ are the nonzero distinct eigenvalues of $\mathbf{S}_{k}$ and $\tilde{\mathbf{S}}_{k}$, respectively. Here, $E_{1}(x)=$ $\int_{1}^{\infty} e^{-x u} / u d u$ is the exponential integral.

Proof: The proof is given in Appendix A.

As stated in Theorem 1, a requirement for the expression in (5) is that all nonzero eigenvalues of $\mathbf{S}_{k}$ and $\tilde{\mathbf{S}}_{k}$ are distinct. In the unlikely event of nondistinct eigenvalues, general expressions can be derived using the theory of [23] and [24].

\section{Characterization OF THE PARETO Boundary}

In this section, we analyze the achievable rate region for the scenario at hand, which will provide a precoding structure that is used for practical precoding design in Section IV. Since the receivers are assumed to treat co-channel interference as noise (i.e., not attempting to decode and subtract the interference), the achievable rate region will in general be smaller than the information theoretic capacity region. This limiting assumption is however relevant in the case of simple receiver structures. In the case of instantaneous CSI, we define the achievable rate region as

$$
\mathcal{R}_{\text {instant }}=\bigcup_{\substack{\left\{\mathbf{w}_{j k} \in \mathbb{C}^{N_{t}} \\\left\{p_{j k} \forall j, k ; p_{j k} \geq 0, \sum_{k=1}^{K_{r}} p_{j k} \leq P_{j}\right\}\right.}}\left(R_{1}, \ldots, R_{K_{r}}\right)
$$

while in the case of statistical CSI we define the achievable expected rate region as

$$
\mathcal{R}_{\text {statistic }}=\bigcup_{\substack{\left\{\mathbf{w}_{j k} \in \mathbb{C}^{N_{t}} \\\left\{p_{j k} \forall j, k ; p_{j k} \geq 0, \sum_{k=1}^{K_{r}} p_{j k} \leq P_{j}\right\}\right.}}\left(\mathbb{E}\left\{R_{1}\right\}, \ldots, \mathbb{E}\left\{R_{K_{r}}\right\}\right) .
$$

Observe that all rates are functions of all $\mathbf{w}_{j k}$ and $p_{j k}$, although not written explicitly. The above rate regions characterize, respectively, all rate tuples $\left(R_{1}, \ldots, R_{K_{r}}\right)$ and expected rate tuples $\left(\mathbb{E}\left\{R_{1}\right\}, \ldots, \mathbb{E}\left\{R_{K_{r}}\right\}\right)$ that are achievable with feasible precoding strategies, regardless of how these strategies are obtained. Our assumption of local CSI at the transmitters determines which rate tuples can be reached by practical algorithmic selection of $\mathbf{w}_{j k}$ and $p_{j k}$, since it restricts the latter to be functions of the local knowledge alone, as opposed to being functions of the whole channel knowledge as traditionally assumed (see Section IV).

The outer boundary of $\mathcal{R}$ is known as the Pareto boundary. The rate tuples on this boundary are Pareto optimal, which 
means that the rate achieved by $\mathrm{MS}_{k}$ cannot be increased without decreasing the rate of any of the other receivers. Each Pareto optimal rate tuple maximizes a certain weighted sum rate. We have the following definition of the Pareto boundary in the case of instantaneous CSI.

Definition 1: Consider all achievable rate tuples $\left(R_{1}, \ldots, R_{K_{r}}\right)$. The Pareto boundary consists of all such tuples for which there exist no nonidentical achievable rate tuple $\left(S_{1}, \ldots, S_{K_{r}}\right)$ with $S_{k} \geq R_{k}$ for all $k=1, \ldots, K_{r}$.

The corresponding definition with statistical CSI is achieved by replacing all rates by their expectations. Next, we will parameterize the Pareto boundary of $\mathcal{R}_{\text {instant }}$ by showing that beamformers, $\mathbf{w}_{j k}$, that can be used to attain the boundary lie in a certain subspace defined by only local CSI and that full transmit power $\left(\sum_{k=1}^{K_{r}} p_{j k}=P_{j}\right)$ should be used by all base stations. In Section III-B, we derive a similar characterization of the Pareto boundary of $\mathcal{R}_{\text {statistic }}$ for systems with statistical CSI.

\section{A. Characterization With Instantaneous CSI}

Two classic beamforming strategies are maximum ratio combining (MRT) and zero-forcing (ZF), which maximizes the received signal power and minimizes the coterminal interference, respectively. In the special case of $K_{r}=2$, these beamforming vectors are aligned with $\mathbf{h}_{j k}$ and $\Pi_{\mathbf{h}_{j \bar{k}}}^{\perp} \mathbf{h}_{j k}$, respectively, for $\bar{k} \neq k$. It was shown in [14] that the Pareto boundary of the MISO IC and BC with $K_{t}=K_{r}=2$ can only be attained by beamformers that are linear combinations of MRT and ZF. This optimal strategy is interesting from a game theoretical perspective, since it can be interpreted as a combination of the selfish MRT and the altruistic ZF approach.

The system defined in Section II represents cooperative multicell precoding with data sharing. This scenario is fundamentally different from the MISO IC as the data sharing enables terminals to be served by multiple transmitters, and thus the achievable rate region can be considerably larger. The following theorem derives the optimal precoding characterization for this scenario, which turns out to be a conceptually similar combination of MRT and ZF. It also constitutes a novel extension to an arbitrary number of transmitters/receivers.

Theorem 2: For each rate tuple $\left(R_{1}, \ldots, R_{K_{r}}\right)$ on the Pareto boundary it holds that

i) It can be achieved by beamformers $\mathbf{w}_{j k}$ that fulfill

$$
\mathbf{w}_{j k} \in \operatorname{span}\left(\left\{\mathbf{h}_{j k}\right\} \bigcup_{\bar{k} \neq k}\left\{\boldsymbol{\Pi}_{\mathbf{h}_{j k}}^{\perp} \mathbf{h}_{j k}\right\}\right) \quad \text { for all } j, k .
$$

ii) If $\mathbf{h}_{j k} \notin \operatorname{span}\left(\bigcup_{\bar{k} \neq k}\left\{\mathbf{h}_{j \bar{k}}\right\}\right)$ for some $k$, then a necessary condition for Pareto optimality is that $\mathrm{BS}_{j}$ uses full power (i.e., $\sum_{k} p_{j k}=P_{j}$ ) and selects $\mathbf{w}_{j k}$ that satisfy (8) for all $k$. Proof: The proof is given in Appendix A.

The theorem implies that to attain rate tuples on the Pareto boundary, all transmitters are required to use full transmit power (except in a special case with zero probability) and use beamforming vectors that can be expressed as

$$
\mathbf{w}_{j k}=\gamma_{j k}^{(k)} \mathbf{h}_{j k}+\sum_{\substack{k=1 \\ k \neq k}}^{K_{r}} \gamma_{j k}^{(\bar{k})} \Pi_{\mathbf{h}_{j \bar{k}}}^{\perp} \mathbf{h}_{j k}, \quad \text { for all } j, k,
$$

for some coefficients $\gamma_{j k}^{(1)}, \ldots, \gamma_{j k}^{\left(K_{r}\right)} \in \mathbb{C}$. This is a linear combination of $K_{r}-1$ zero-forcing vectors $\Pi_{\mathbf{h}_{j \bar{k}}}^{\perp} \mathbf{h}_{j k}$ (each inflicting zero interference at $\mathrm{MS}_{\bar{k}}$ ) and the following MRT beamformer:

Definition 2 (Maximum Ratio Transmission):

$$
\mathbf{w}_{j k}^{(\mathrm{MRT})}=\frac{\mathbf{h}_{j k}}{\left\|\mathbf{h}_{j k}\right\|} \quad \text { for all } j, k .
$$

Complete ZF beamforming that inflicts zero interference on all co-terminals exists if $N_{t} \geq K_{r}$ and can be defined in the following way, but observe that it can also be expressed as the linear combination in (9).

$$
\text { Definition } 3 \text { (Zero-Forcing Beamforming): If } N_{t} \geq K_{r} \text {, }
$$

$$
\mathbf{w}_{j k}^{(\mathrm{ZF})}=\frac{\left(\mathbf{I}-\sum_{l=1}^{m_{j k}} \boldsymbol{\Pi}_{\mathbf{e}_{j k}^{(l)}}\right) \mathbf{h}_{j k}}{\left\|\left(\mathbf{I}-\sum_{l=1}^{m_{j k}} \boldsymbol{\Pi}_{\mathbf{e}_{j k}^{(l)}}\right) \mathbf{h}_{j k}\right\|}
$$

where $\mathbf{e}_{j k}^{(1)}, \ldots, \mathbf{e}_{j k}^{\left(m_{j k}\right)}$ is an orthogonal basis of $\operatorname{span}\left(\bigcup_{\bar{k} \neq k}\left\{\mathbf{h}_{j \bar{k}}\right\}\right)$, for all $j, k$.

Several important conclusions can be drawn from the theorem. First, the precoding characterization reduces the precoding complexity if $N_{t}>K_{r}$ (since the beamforming vectors we are looking for each lie in a $K_{r}$-dimensional subspace ${ }^{3}$ ), especially if $N_{t}$ is large. Second, the optimal beamforming approach can be interpreted as a linear combination of the selfish MRT approach and altruistic interference control towards each co-terminal. This behavior has been pointed out in [14] for the MISO IC, but not for multicell precoding systems. Third, the characterization is defined using only local CSI, while global information is required to find the optimal coefficients $\gamma_{j k}^{(\bar{k})}$. In Section IV, we discuss heuristic approaches for distributed computation of the coefficients and evaluate their performance in Section V. Apart from selecting beamforming vectors, it is necessary to perform optimal power allocation to attain the Pareto boundary. Some power allocation strategies that exploit local CSI are also provided in Section IV.

\section{B. Characterization With Statistical CSI}

Next, we characterize the Pareto boundary in a similar manner as in Theorem 2, but for the case of statistical CSI. It was shown in [25], for the MISO IC, that an exact parametrization can be derived when the correlation matrices are rank deficient. This is however rarely the case in practice and therefore we concentrate on general spatially correlated channels and characterize their correlation matrices, $\mathbf{Q}_{j k}$. Depending on the antenna distance and the amount of scattering, the channels from transmit antennas to the receiver have varying spatial correlation; large antenna spacing and rich scattering correspond to low spatial correlation, and vice versa. High correlation translates into large eigenvalue spread in $\mathbf{Q}_{j k}$ and low correlation to almost identical eigenvalues. The existence of strongly structured spatial correlation has been verified experimentally, in both outdoor [26] and indoor [27] scenarios,

\footnotetext{
${ }^{3}$ In practice, this dimension can be further reduced by ignoring inactive receivers and those with negligible link gains to the transmitter.
} 
and we will show herein how to exploit these results in the context of multicell precoding. In particular, these results suggest the existence of a dominating subspace.

Similar to [28], we partition the eigenvalue decomposition $\mathbf{Q}_{j k}=\mathbf{U}_{j k} \boldsymbol{\Lambda}_{j k} \mathbf{U}_{j k}^{H}$ of the correlation matrix $\mathbf{Q}_{j k}$ in signal and interference subspaces based on the size of the eigenvalues. Assume that the eigenvalues in the diagonal matrix $\boldsymbol{\Lambda}_{j k}$ are ordered decreasingly with the corresponding eigenvectors as columns of the unitary matrix $\mathbf{U}_{j k}$. Then, we partition $\mathbf{U}_{j k}$ as

$$
\mathbf{U}_{j k}=\left[\mathbf{U}_{j k}^{(D)} \mathbf{U}_{j k}^{(0)}\right]
$$

where $\mathbf{U}_{j k}^{(D)} \in \mathbb{C}^{N_{t} \times N_{d}}$ spans the subspace associated with the $N_{d} \leq N_{t}$ dominating eigenvalues. The transmit power allocated to these eigendirections will have large impact on the SINR. Consequently, data transmission should take place in the range of eigendirections included in $\mathbf{U}_{j k}^{(D)}$, while one should avoid receiving interference in these directions. Assuming that the nondominating eigenvalues associated with the remaining eigenvectors in $\mathbf{U}_{j k}^{(0)} \in \mathbb{C}^{N_{t} \times N_{t}-N_{d}}$ are much smaller than the dominating ones, the interference in this subspace will be limited. The design parameter $N_{d}$ depends strongly on the amount of spatial correlation, and can be a small fraction of $N_{t}$ in an outdoor cellular scenario. In completely uncorrelated environments, the partitioning can be ignored since $N_{d}=N_{t}$. Feedback of instantaneous channel norms and receive beamforming (in the case of multi-antenna receivers) can increase the effective spatial correlation and thereby decrease $N_{d}$ [23]. In practice, careful measurements are necessary to determine the value that maximizes the average throughput.

Now, we will characterize the Pareto boundary of the achievable expected rate region for cooperative multicell precoding. It will be done in an approximate manner, using the eigenvector partitioning in (10). The following theorem is more general than its counterpart for the MISO IC in [25] as it considers an arbitrary number of transmitters/receivers and correlation matrices of full rank.

Theorem 3: Let the sum of nondominating eigenvalues in $\mathbf{Q}_{1 k}, \ldots, \mathbf{Q}_{K_{t} k}$ be denoted $\epsilon_{k} \triangleq \sum_{j=1}^{K_{t}} \operatorname{tr}\left(\left(\mathbf{U}_{j k}^{(0)}\right)^{H} \mathbf{Q}_{j k} \mathbf{U}_{j k}^{(0)}\right)$, for all $k$. For each expected rate tuple $\left(\mathbb{E}\left\{R_{1}\right\}, \ldots, \mathbb{E}\left\{R_{K_{r}}\right\}\right)$ on the Pareto boundary, there exists with probability one another achievable tuple $\left(\mathbb{E}\left\{\tilde{R}_{1}\right\}, \ldots, \mathbb{E}\left\{\tilde{R}_{K_{r}}\right\}\right)$ that fulfills $\mathbb{E}\left\{R_{k}\right\}=$ $\mathbb{E}\left\{\tilde{R}_{k}\right\}+o\left(\epsilon_{k}\right)$ for all $k$, where the small $o$ function means that $\mathbb{E}\left\{R_{k}\right\}-\mathbb{E}\left\{\widetilde{R}_{k}\right\} \rightarrow 0$ as $\epsilon_{k} \rightarrow 0$. For the rate tuple $\left(\mathbb{E}\left\{\tilde{R}_{1}\right\}, \ldots, \mathbb{E}\left\{\tilde{R}_{K_{r}}\right\}\right)$ it holds that

1) It can be reached using beamforming vectors

$$
\mathbf{w}_{j k} \in \operatorname{span}\left(\left\{\mathbf{U}_{j k}^{(D)}\right\} \bigcup_{\bar{k} \neq k}\left\{\boldsymbol{\Pi}_{\mathbf{U}_{j k}^{(D)}}^{\perp} \mathbf{U}_{j k}^{(D)}\right\}\right){ }_{\text {for all } j, k ;}
$$

2) If $\operatorname{span}\left(\bigcup_{k=1}^{K_{r}}\left\{\mathbf{U}_{j k}^{(D)}\right\}\right) \neq \mathbb{C}^{N_{t}}$ for some $j$, it can be reached when $\mathrm{BS}_{j}$ uses full power.

Proof: The proof is given in Appendix A.

Observe that in the special case of zero-valued eigenvalues within each nondominating eigenspace $\mathbf{U}_{j k}^{(0)}$ (i.e., $\epsilon_{k}=0$ ), the theorem gives an exact characterization since $\mathbb{E}\left\{R_{k}\right\}=$ $\mathbb{E}\left\{\tilde{R}_{k}\right\}$.

There are clear similarities between the precoding characterization in (8) for instantaneous CSI, and its counterpart in (11) for statistical CSI. In both cases, all interesting beamforming vectors are linear combinations of eigenvectors that (selfishly) provide strong signal gain and that (altruistically) limit the interference at coterminals. These eigenvectors are defined using local CSI, which enables distributed precoding in a structured manner (see Section IV). The results with statistical CSI are however weaker, which is natural since each channel vector belongs (approximately) to a subspace of rank $N_{d}$ while the channels with instantaneous CSI are known vectors (i.e., rank one). In the special case of $N_{d}=1$, the characterization in Theorem 3 becomes essentially the same as in Theorem 2 .

From these observations, it is natural to consider the two extremes that satisfy the beamforming characterization, namely MRT and ZF. Analogously to the MRT and ZF approaches with instantaneous CSI in Definitions 2 and 3, we propose extensions to the case of statistical CSI. The straightforward generalization of MRT is to use the dominating eigenvector of $\mathbf{Q}_{j k}$ as beamformer in $\mathbf{w}_{j k}$. We denote the normalized eigenvector associated with the largest eigenvalue of $\mathbf{Q}_{j k}$ by $\mathbf{u}_{j k}^{(D)}$. The generalization of $\mathrm{ZF}$ is to maximize the average received signal power under the condition that the beamformer lies in the nondominating eigen-subspace $\mathbf{U}_{j \bar{k}}^{(0)}$ of all coterminals. Formally, we have the following precoding approaches.

Definition 4 (Generalized MRT):

$$
\mathbf{w}_{j k}^{(\mathrm{G}-\mathrm{MRT})}=\mathbf{u}_{j k}^{(D)} \quad \text { for all } j, k .
$$

Definition 5 (Generalized ZF):

$$
\mathbf{w}_{j k}^{(\mathrm{G}-\mathrm{ZF})}=\mathbf{v}_{j k}
$$

where $\mathbf{v}_{j k}$ is the normalized dominating eigenvector of $\boldsymbol{\Pi}_{\mathcal{S}_{j k}}^{\perp} \mathbf{Q}_{j k} \boldsymbol{\Pi}_{\mathcal{S}_{j k}}^{\perp}$ with $\mathcal{S}_{j k}=\operatorname{span}\left(\bigcup_{\bar{k} \neq k}\left\{\mathbf{U}_{j \bar{k}}^{(D)}\right\}\right)$, for all $j, k$.

Observe that generalized $\mathrm{ZF}$ only exists for certain combinations of $N_{d}$ and $K_{r}$ as it is necessary that $\operatorname{rank}\left(\mathcal{S}_{j k}\right)<N_{t}$. The generalizations in Definition 4 and 5 are made for multicell precoding. For broadcast channels, and in general, other generalizations are possible.

\section{Distributed Precoding With Local CSI}

In the previous section, we characterized the beamforming vectors that can be used to attain the Pareto boundary of the achievable rate region. These are all linear combinations of MRT and ZF, the two extremes in beamforming. Intuitively, MRT is the asymptotically optimal strategy at low SNR, while ZF works well at high SNR or as the number of antennas increases. In general, the optimal strategy lies in between these extremes and cannot be determined without global CSI. Next, we use these insights to solve distributed precoding at an arbitrary SNR using only local CSI. The proposed beamforming approach is inspired by uplink-downlink duality for broadcast channels [19] and the transmit power is allocated heuristically by solving local optimization problems. The approach is asymptotically optimal at high SNR and the numerical evaluation in Section V shows a limited performance loss at all SNRs. 


\section{A. Transmission Design With Local Instantaneous CSI}

In general, we would like the precoding to solve

$$
\underset{\mathbf{w}_{j k} \in \mathbb{C}^{N_{t}}, p_{j k} \geq 0}{\operatorname{maximize}} \sum_{\forall j, k}^{K_{r}} \log _{2}\left(1+\operatorname{SINR}_{k}\right)
$$

subject to $\left\|\mathbf{w}_{j k}\right\|=1$ and $\sum_{k=1}^{K_{r}} p_{j k} \leq P_{j}$ for all $j$ and $k$. Unfortunately, none of the transmitters or receivers have sufficient CSI to calculate the sum rate, which makes the optimization problem in (12) intractable in a truly distributed scenario. Thus, we will look for distributed design criteria that allow approximated beamforming vectors, $\mathbf{w}_{j k}$, and power allocation coefficients, $p_{j k}$, of $\mathrm{BS}_{j}$ to be determined locally at the transmitter. The goal will still be to achieve performance close to the maximum sum rate. An important feature of the precoding characterization in Theorem 2 is that the optimal $\mathbf{w}_{j k}$ fulfills

$$
\mathbf{w}_{j k} \in \operatorname{span}\left(\left\{\mathbf{h}_{j k}\right\} \bigcup_{\bar{k} \neq k}\left\{\boldsymbol{\Pi}_{\mathbf{h}_{j k}}^{\perp} \mathbf{h}_{j k}\right\}\right)
$$

where all the spanning vectors are known locally at $\mathrm{BS}_{j}$. In other words, the beamforming design consists of determining the coefficients of the linear combination in (9). To find heuristic coefficients, we exploit the following result based on the uplinkdownlink duality theory of [19].

Theorem 4: Assume that $\mathrm{BS}_{j}$ is the only active base station. Then, each Pareto optimal rate tuple of the corresponding achievable rate region is achieved by beamforming vectors

$$
\mathbf{w}_{j k}=\underset{\|\mathbf{w}\|^{2}=1}{\arg \max } \frac{\beta_{j k}\left|\mathbf{h}_{j k}^{H} \mathbf{w}\right|^{2}}{\frac{\sigma_{k}^{2}}{P_{j}}+\sum_{\bar{k} \neq k} \beta_{j \bar{k}}\left|\mathbf{h}_{j \bar{k}}^{H} \mathbf{w}\right|^{2}}
$$

for some positive coefficients $\beta_{j k}$ with $\sum_{k=1}^{K_{r}} \beta_{j k}=1$.

Proof: The proof is given in Appendix A.

Thus, in the special case of a MISO broadcast channel, the optimal beamforming vectors are achieved by maximizing the SINR-like expression in (14) where the signal power that $\mathrm{BS}_{j}$ generates at $\mathrm{MS}_{k}$ is balanced against the noise and interference power generated at all other receivers. We call it a virtual SINR as it originates from the dual virtual uplink [19] and does not directly represent the SINR of any of the links in the downlink. However, it is easy to show ${ }^{4}$ that solutions to (14) are of the type described in (9) and (13). In fact, by varying the coefficients $\beta_{j k}$, different solutions within the span of Theorem 2 can be achieved. In general, the coefficients that provides the largest sum rate can only be found using global CSI.

Network MIMO can be seen as a superposition of $K_{t}$ broadcast channels. We propose to exploit this fact for distributed precoding by letting each base station optimize its performance based on Theorem 4. In the superposition case, the noise term of (14) should be modified to compensate for the interference from other base stations, or equivalently the coefficients $\beta_{j k}$ should be increased beyond what is allowed for pure broadcast channels. To account for stronger interference we therefore select

\footnotetext{
${ }^{4}$ Observe that $\mathbf{w}$ should lie in the span of $\mathbf{h}_{j k}$, for all $k$, as no other directions will affect (14). We achieve (13) by rewriting this span following the approach in the proof of Theorem 2 .
}

$\beta_{j k}=1$ (i.e., equal to its upper bound) and arrive at a novel distributed virtual SINR (DVSINR) beamforming approach:

Strategy 1: $\mathrm{BS}_{j}$ should select its beamformers as

$$
\mathbf{w}_{j k}^{(\text {DVSINR })}=\underset{\|\mathbf{w}\|^{2}=1}{\arg \max } \frac{\left|\mathbf{h}_{j k}^{H} \mathbf{w}\right|^{2}}{\frac{\sigma_{k}^{2}}{P_{j}}+\sum_{\bar{k} \neq k}\left|\mathbf{h}_{j \bar{k}}^{H} \mathbf{w}\right|^{2}} \text { for all } k .
$$

Observe that the virtual SINR in (15) is a Rayleigh quotient and thus the maximization can be solved by straightforward eigenvalue techniques. For example

$$
\begin{aligned}
\mathbf{w}_{j k}^{(\text {DVSINR })} & =\frac{\mathbf{C}_{j k}^{-1} \mathbf{h}_{j k}}{\left\|\mathbf{C}_{j k}^{-1} \mathbf{h}_{j k}\right\|} \text { where } \\
\mathbf{C}_{j k} & \triangleq \frac{\sigma_{k}^{2}}{P_{j}} \mathbf{I}+\sum_{\bar{k} \neq k} \mathbf{h}_{j \bar{k}} \mathbf{h}_{j \bar{k}}^{H} .
\end{aligned}
$$

The solution to (15) is nonunique, since the virtual SINR is unaffected by phase shifts in w. However, the expression above was selected to make $\mathbf{h}_{j k}^{H} \mathbf{w}$ positive and real-valued, which means that the signals arriving at a given terminal from different base stations will do so constructively. By its very definition, maximization of a virtual SINR effectively balances between the useful signal power at a target terminal and the interference generated at others; along with judicious power allocation coefficients $p_{j k}$ for all $j, k$, this can be shown to provide good performance at all SNRs (see Section V). Observe that (15) gives solutions similar to MRT and ZF in the SNR regimes where these methods perform well (i.e., low SNR and high SNR, respectively). Asymptotic optimality conditions are provided by the following theorem.

Theorem 5: Assume an arbitrary power allocation which guarantees that each terminal is allocated nonzero total transmit power (i.e., $\sum_{j=1}^{K_{t}} p_{j k}>0$ for all $k$ ). If $N_{t} \geq K_{r}{ }^{5}$ and each $p_{j k}>0$ increases with $P_{j}$, then with probability one DVSINR beamforming achieves the full multiplexing gain of $K_{r}$ asymptotically in $P=\min _{j} P_{j}$.

Proof: The proof is given in Appendix A.

This means that the sum rate behaves as $K_{r} \log _{2}(P)+$ constant at high $P$. Thus, the absolute performance loss compared with optimal centralized precoding is bounded at high SNR and the relative loss goes to zero. The absolute loss is primarily due to the fact that distributed beamforming limits the magnitude of interference from each transmitter to every terminal, while the global solution can coordinate and cancel out the sum of interference from different transmitters. However, such centralized interference coordination is practically questionable even under optimal conditions [29].

The power allocation has a clear impact on the practical performance, although Theorem 5 holds for any allocation. Next, we propose a heuristic power allocation scheme for $\mathrm{BS}_{j}$. This is based on the observation that with proper beamforming, the interference is negligible at both low and high SNR. Assuming

\footnotetext{
${ }^{5}$ The constraint $N_{t} \geq K_{r}$ can be removed if each $\mathrm{BS}_{j}$ have nonnegligible channel gain to at most $N_{t}$ terminals.
} 
constructive signal contributions from all base stations, the sum rate then becomes

$$
\begin{aligned}
& \sum_{k=1}^{K_{r}} \log _{2}\left(1+\operatorname{SINR}_{k}\right) \\
& \approx \sum_{k=1}^{K_{r}} \log _{2}\left(1+|\sqrt{p_{j k}} \underbrace{\frac{\mathbf{h}_{j k}^{H} \mathbf{w}_{j k}}{\sigma_{k}}}_{=c_{j k}}+\underbrace{\sum_{\bar{j} \neq j} \sqrt{p_{\bar{j} k}} \frac{\mathbf{h}_{\bar{j} k}^{H} \mathbf{w}_{\bar{j} k}}{\sigma_{k}}}_{=d_{j k}}|^{2}\right)
\end{aligned}
$$

where $\left|c_{j k}\right|^{2}$ denotes the channel gain between $\mathrm{BS}_{j}$ and $\mathrm{MS}_{k}$ and $\left|d_{j k}\right|^{2}$ is the signal gain from the other transmitters (including power allocation). All $c_{j k}$ and $d_{j k}$ can be taken as positive real-valued, due to the assumption of transmission synchronization. For fixed values on all $c_{j k}$ and $d_{j k}$, the power allocation at $\mathrm{BS}_{j}$ is solved by the following lemma.

Lemma 1: For a given $j$ and some positive constants $c_{j k}, d_{j k}$, the optimization problem

$$
\begin{aligned}
\underset{p_{j 1} \geq 0, \ldots, p_{j K_{r}} \geq 0}{\operatorname{maximize}} & \sum_{k=1}^{K_{r}} \log _{2}\left(1+\left(\sqrt{p_{j k}} c_{j k}+d_{j k}\right)^{2}\right) \\
\text { subject to } & \sum_{k=1}^{K_{r}} p_{j k} \leq P_{j}
\end{aligned}
$$

is solved by

$$
\begin{aligned}
\sqrt{p_{j k}}= & \max \left(\sqrt[3]{a_{j k}+\sqrt{a_{j k}^{2}+b_{j k}^{3}}}\right. \\
& \left.-\frac{b_{j k}}{\sqrt[3]{a_{j k}+\sqrt{a_{j k}^{2}+b_{j k}^{3}}}}-\frac{2 d_{j k}}{3 c_{j k}}, 0\right), \\
a_{j k}= & \frac{18 d_{j k}+2 d_{j k}^{3}+9 \alpha c_{j k}^{2} d_{j k}}{54 c_{j k}^{3}}, \\
b_{j k}= & \frac{3-d_{j k}^{2}}{9 c_{j k}^{2}}-\frac{\alpha}{3} .
\end{aligned}
$$

If $d_{j k}=0$, this reduces to $\sqrt{p_{j k}}=\max \left(\alpha-1 / c_{j k}^{2}, 0\right)$. The Lagrange multiplier $\alpha \geq 0$ is selected to fulfill the power constraint with equality.

Proof: The maximization of a concave function can be solved by standard Lagrangian methods, using the Karush-Kuhn-Tucker (KKT) conditions ([30, Ch. 5.5]). In this case, the optimal power allocation follows from straightforward differentiation, solving of a third-order polynomial equation with respect to $\sqrt{p_{j k}}$, and identifying the two false roots.

The local channel gains $c_{j k}$ are known at each $\mathrm{BS}_{j}$, while the contributions $d_{j k}$ from other transmitters are unknown when having local CSI. Thus, $\mathrm{BS}_{j}$ needs to estimate these parameters. To avoid that all transmitters believe that someone else serves a given terminal, the estimate should be pessimistic. In a symmetric environment, the selection

$$
d_{j k}^{\mathrm{symmetric}}=\sqrt{\frac{P_{j}}{N_{t}} \sum_{\bar{k}=1}^{K_{r}} \frac{\left|\mathbf{h}_{j \bar{k}}^{H} \mathbf{w}_{j \bar{k}}\right|^{2}}{K_{r} \sigma_{\bar{k}}^{2}}}
$$

represents that one other transmitter uses $1 / N_{t}$ of its power to serve the terminal (the channel gain is estimated as the average gain from $\mathrm{BS}_{j}$ to all terminals). In other cases, the worst-case selection

$$
d_{j k}^{\text {worst-case }}=0
$$

can give better performance or robustness. In practice, $d_{j k}$ should be considered design parameters and tuned based on measured properties of the actual propagation environment. For given $d_{j k}$ and beamforming vectors $\mathbf{w}_{j k}$, we use Lemma 1 to propose the following power allocation scheme.

Strategy 2: Using local CSI, an efficient power allocation at $\mathrm{BS}_{j}$ for $N_{t} \geq K_{r}$ is given by Lemma 1 using $c_{j k}=\left|\mathbf{h}_{j k}^{H} \mathbf{w}_{j k}\right| / \sigma_{k}$ and some $d_{j k}$ that reflects the propagation environment.

In the case $N_{t}<K_{r}$, the interference can in general not be considered negligible as was assumed in the heuristic power allocation. Thus, alternative power allocation schemes should be considered-for example, the simple scheme $p_{j k}=P_{j}\left\|\mathbf{h}_{j k}\right\|^{2} / \sum_{j}\left\|\mathbf{h}_{j k}\right\|^{2}$ evaluated in [20] and [21].

Observe that the power allocation in Strategy 2 has the waterfilling behavior, which means that zero power is allocated to weak terminals. Thus, terminals far from the base station are disregarded automatically, which limits the computational complexity as $K_{t}$ and $K_{r}$ increases.

\section{B. Transmission Design With Local Statistical CSI}

Next, we extend the precoding design in the previous subsection to the case of local statistical CSI. As in the previous case, maximizing a virtual SINR will balance the generated signal and interference powers. We propose the following novel extension where the Rayleigh quotient represents maximization of an SINR where expectation has been applied to the numerator and denominator (using that $\mathbb{E}\left\{\left|\mathbf{h}_{j k}^{H} \mathbf{w}\right|^{2}\right\}=\mathbf{w}^{H} \mathbf{Q}_{j k} \mathbf{w}$ ).

Strategy 3: For given power allocation coefficients, $\mathrm{BS}_{j}$ should select its beamformers as

$$
\begin{aligned}
& \mathbf{w}_{j k}^{(\mathrm{G}-\mathrm{DVSINR})}=\underset{\|\mathbf{w}\|^{2}=1}{\arg \max } \frac{\mathbf{w}^{H} \mathbf{Q}_{j k} \mathbf{w}}{\frac{\sigma_{k}^{2}}{P_{j}}+\sum_{\bar{k} \neq k} \mathbf{w}^{H} \mathbf{Q}_{j \bar{k}} \mathbf{w}} \\
& \text { for all } k \text {. }
\end{aligned}
$$

Unlike the case of instantaneous CSI, beamforming design with statistical CSI cannot guarantee coherent arrival of useful signals at a given receiver, but an increase in signal power will improve the average rate. The distributed SINR beamforming vectors of (21) satisfy (approximately) the beamforming characterization in Theorem 3.

Finally, we derive a distributed power allocation scheme. Since the expected rate expression in (5) is complicated, we 
simplify it by neglecting the interference. For $\mathrm{MS}_{k}$, the expected rate in Theorem 1 becomes

$$
\begin{aligned}
& \mathbb{E}\left\{\log _{2}\left(1+\operatorname{SINR}_{k}\right)\right\} \\
& \approx \frac{e^{\sigma_{k}^{2} / \mu_{1}} E_{1}\left(\sigma_{k}^{2} / \mu_{1}\right)}{\log (2)} \\
& \approx \log _{2}(1+p_{j k} \underbrace{\frac{\mathbf{w}_{j k}^{H} \mathbf{Q}_{j k} \mathbf{w}_{j k}}{\sigma_{k}^{2}}}_{=f_{j k}}+\underbrace{\sum_{\bar{j} \neq j} \frac{p_{\bar{j} k} \mathbf{w}_{\bar{j} k}^{H} \mathbf{Q}_{\bar{j} k} \mathbf{w}_{\bar{j} k}}{\sigma_{k}^{2}}}_{=g_{j k}})
\end{aligned}
$$

using the upper part of the bound $(1 / 2) \log \left(1+2 \mu_{1} / \sigma_{k}^{2}\right)<$ $e^{\sigma_{k}^{2} / \mu_{1}} E_{1}\left(\sigma_{k}^{2} / \mu_{1}\right)<\log \left(1+\mu_{1} / \sigma_{k}^{2}\right)$. Here, $f_{j k}$ denotes the average channel gain between $\mathrm{BS}_{j}$ and $\mathrm{MS}_{k}$ and $g_{j k}$ is an estimation of the average signal gain from the other transmitters (including power allocation). For fixed values on all $g_{j k}$, the power allocation at $\mathrm{BS}_{j}$ is solved by the following lemma.

Lemma 2: For a given $j$ and some positive $f_{j k}, g_{j k}$, the optimization problem

$$
\begin{array}{ll}
\text { maximize } & \sum_{k=1}^{K_{r}} \log _{2}\left(1+p_{j k} f_{j k}+g_{j k}\right) \\
\text { subject to } & \sum_{k=1}^{K_{r}} p_{j k} \leq P_{j}, p_{j 1} \geq 0, \ldots, p_{j K_{r}} \geq 0
\end{array}
$$

is solved by $p_{j k}=\max \left(\alpha-\left(1+g_{j k}\right) / f_{j k}, 0\right)$, where the Lagrange multiplier $\alpha \geq 0$ is selected to fulfill the power constraint with equality.

Proof: The solution to this convex optimization problem follows from straightforward Lagrangian methods, see the proof of Lemma 1 for details.

Using only local statistical CSI, the average local channel gains $f_{j k}$ are known at $\mathrm{BS}_{j}$, while the contributions $g_{j k}$ from other transmitters are unknown. Thus, $\mathrm{BS}_{j}$ needs to estimate these parameters, which can be done similarly to (19) and (20):

$$
\begin{aligned}
g_{j k}^{\text {symmetric }} & =\frac{P_{j}}{N_{t}} \sum_{\bar{k}=1}^{K_{r}} \frac{\mathbf{w}_{j \bar{k}}^{H} \mathbf{Q}_{j \bar{k}} \mathbf{w}_{j \bar{k}}}{K_{r} \sigma_{\bar{k}}^{2}} \\
g_{j k}^{\text {worst-case }} & =0 .
\end{aligned}
$$

For given $g_{j k}$ and beamforming vectors $\mathbf{w}_{j k}$, we use Lemma 2 to propose the following power allocation scheme.

Strategy 4: Using local CSI, an efficient power allocation at $\mathrm{BS}_{j}$ is given by Lemma 2 using $f_{j k}=\mathbf{w}_{j k}^{H} \mathbf{Q}_{j k} \mathbf{w}_{j k} / \sigma_{k}^{2}$ and some $g_{j k}$ that reflects the propagation environment.

\section{NUMERICAL EXAMPLES}

In this section, the performance of the distributed beamforming and power allocation strategies in Section IV will be illustrated numerically. The DVSINR approach in Strategy 1 will be compared with what we call distributed MRT and distributed $Z F$. These two approaches use the beamforming vectors in Definition 2 and 3, respectively. Observe that there are major differences from regular MRT and ZF for broadcast

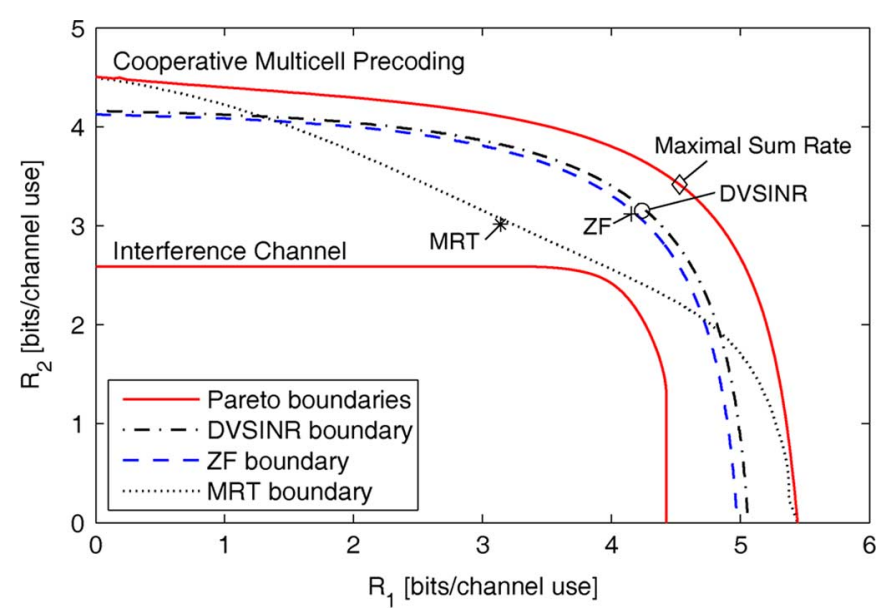

Fig. 2. Achievable rate regions with different beamforming and power allocation for $K_{t}=K_{r}=2, N_{t}=3$, and a random realization with $\mathbf{Q}_{11}=$ $\mathbf{Q}_{22}=\mathbf{I}, \mathbf{Q}_{12}=\mathbf{Q}_{21}=0.5 \mathbf{I}$, and SNR $5 \mathrm{~dB}$. The sum rate point and the points achieved by MRT, ZF, and DVSINR with the power allocation in Strategy 2 are shown for comparison.

and interference channels, namely that the same message is sent from multiple transmitters with individual power constraints. When used, distributed MRT and ZF need to be combined with some power allocation, for example the one proposed in Strategy 2.

\section{A. Transmitter-Receiver Pairs With Varying Crosslinks}

First, consider the case of two transmitter-receiver links where the strengths of the crosslinks are varied. The environment is spatially uncorrelated with $N_{t}=3, \mathbf{Q}_{11}=\mathbf{Q}_{22}=\mathbf{I}$, and $\mathbf{Q}_{12}=\mathbf{Q}_{21}=\beta \mathbf{I}$, where $\beta$ is the average cross link power. This represents a two-cell scenario where $\beta$ determines how close the terminals are to the common cell edge. The SNR is defined as $\mathrm{SNR}=P_{j} \operatorname{tr}\left(\mathbf{Q}_{j j}\right) / N_{t}$ (with normalization $\sigma_{k}^{2}=1$ ) and represents the average SNR for beamforming to the own terminal.

In Fig. 2, the Pareto boundary with $\beta=0.5$ and an average SNR of $5 \mathrm{~dB}$ is given for a random realization of $\mathbf{h}_{j k}$ drawn from $\mathcal{C N}\left(\mathbf{0}, \mathbf{Q}_{j k}\right)$, for all $j, k$. As a comparison, we give the Pareto boundary of the MISO IC [14] and show the outer boundaries of the achievable rate regions with the DVSINR approach in Strategy 1, distributed MRT, and distributed ZF. The rate tuples achieved with the power allocation in Strategy 2 (with $\left.d_{j k}=0\right)$ and the sum rate maximizing point are given as references. For the selected realization there is a clear performance gain of allowing cooperative multicell precoding as compared with forcing each transmitter to only communicate with its own receiver. As expected, MRT is useful to maximize the rate of only one of the terminals, while ZF and DVSINR are quite close to the optimal sum rate point. The proposed power allocation scheme provides performance close to the boundary of each achievable rate region.

In Fig. 3, the average sum rates (over channel realizations) are given with optimal linear precoding (i.e., sum rate maximization through exhaustive search) and with the DVSINR approach in Strategy 1, distributed MRT, and distributed ZF (all three using the power allocation in Strategy 2 with $d_{j k}=0$ to ensure robustness), and the VSINR approach in [17] for the MISO IC. 


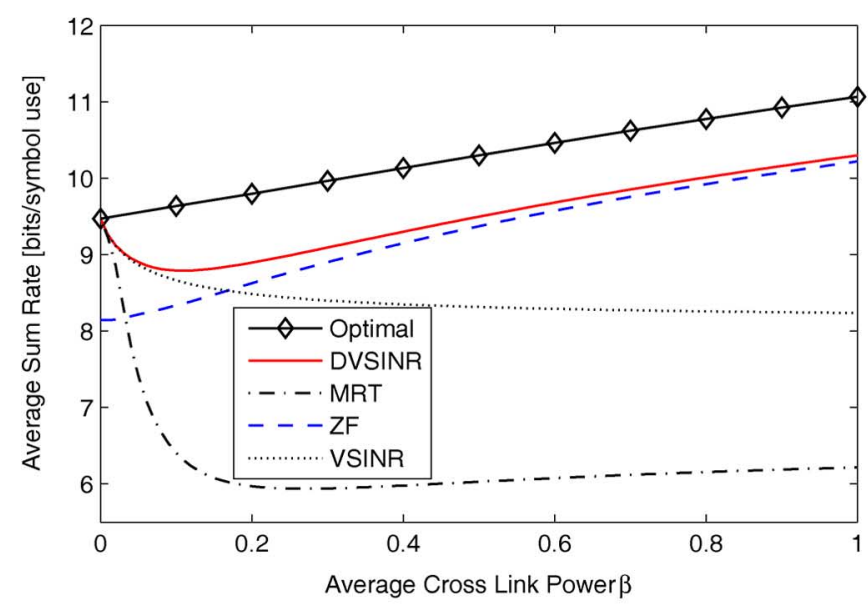

(a)

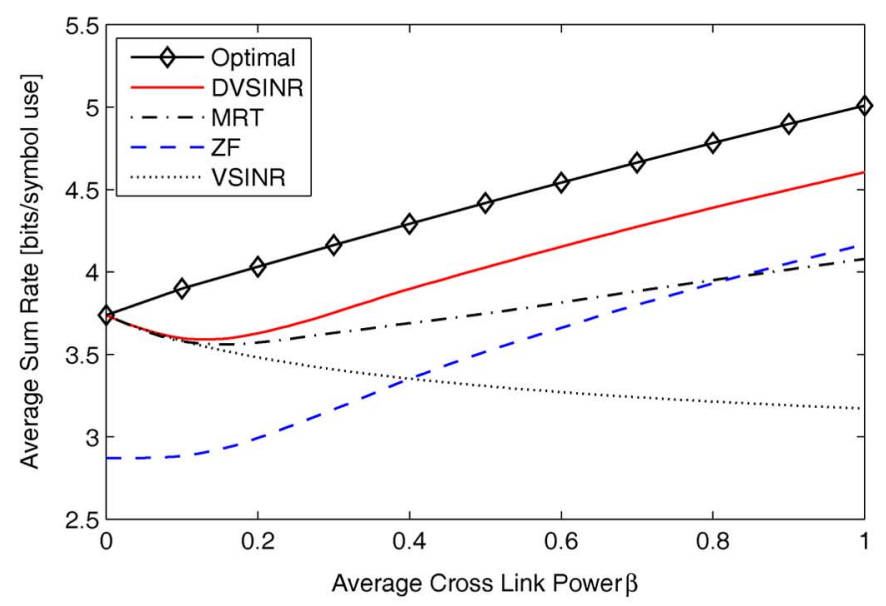

(b)

Fig. 3. Average sum rate (over channel realizations) in a system with local instantaneous CSI, $K_{t}=K_{r}=2, N_{t}=3$, and a varying average cross link power: $\mathbf{Q}_{11}=\mathbf{Q}_{22}=\mathbf{I}, \mathbf{Q}_{12}=\mathbf{Q}_{21}=\beta \mathbf{I}$. (a) SNR $10 \mathrm{~dB}$. (b) SNR $0 \mathrm{~dB}$.

The performance is shown for varying cross link power $\beta$ and at an SNR of 0 or $10 \mathrm{~dB}$. We observe that MRT is good at low SNR and/or weak cross link power, while ZF is better at high SNR and/or strong cross link power. However, the DVSINR approach is the most versatile strategy as it provides higher performance at low SNR and combines the benefits of MRT and $\mathrm{ZF}$ at high SNR. The three cooperative approaches clearly yield better performance than the noncooperative VSINR approach.

In practice, two common terminal locations are close to a base station (i.e., high SNR with weak cross link power) and close to the cell edge (i.e., low SNR with strong crosslink power). From Fig. 3 it is clear that DVSINR is the only of the distributed schemes that provides good performance in both cases, which is an important property as both types of terminals appear simultaneously in practice. Thus, although distributed MRT and ZF achieve performance comparable to DVSINR in special cases, it is fair to say that the DVSINR scheme is the most versatile. Due to the distributed nature of the schemes, there is some performance loss compared with sum rate maximizing precoding. However, we argue that the backhaul and computational demands required to achieve the optimal solution may not be motivated in light of the small performance loss.

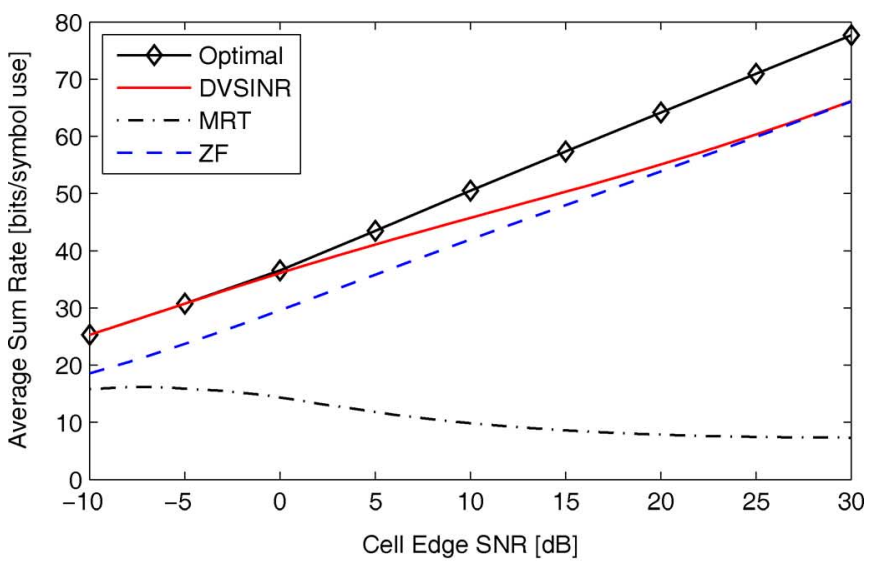

(a)

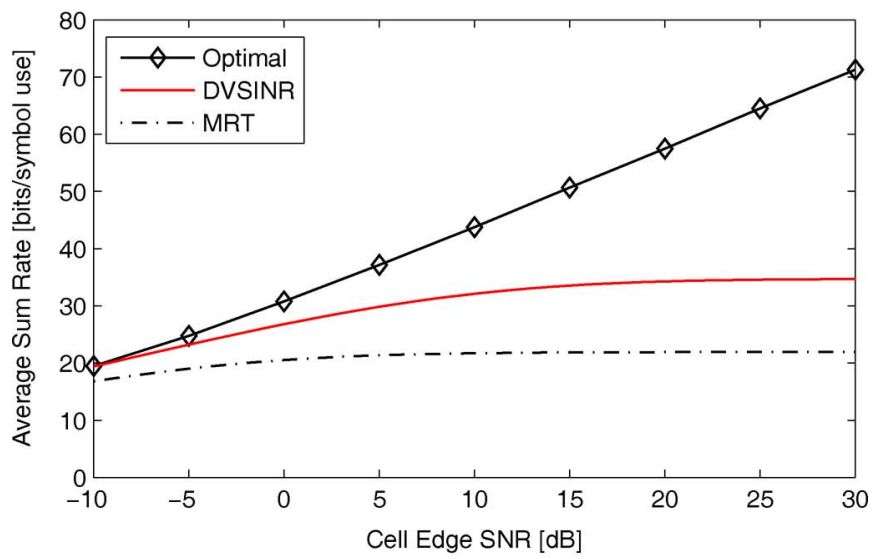

(b)

Fig. 4. Average sum rate (over terminal locations and channel realizations) in a system with local instantaneous CSI and $K_{t}=K_{r}=4$. The scenario considers uniformly distributed terminals within a square with base stations in each corner. (a) $N_{t}=4$. (b) $N_{t}=2$.

\section{B. Quadratic Multicell Area}

Next, we evaluate a scenario with terminals located in both cell centers and at cell edges. The scenario consists of four uniformly distributed terminals in a square with base stations in each of the corners. The power decay is proportional to $1 / r^{4}$, where $r$ is the distance from a transmitter, the SNR is defined as SNR $=P_{j} \operatorname{tr}\left(\mathbf{Q}_{j k}\right) / N_{t}$ (with normalization $\sigma_{k}^{2}=1$ ), and its value in the center of the square represents the cell edge SNR. This represents a scenario where terminals are moving around in the area covered by four base stations. We will illustrate the performance with both instantaneous and statistical CSI.

In Fig. 4, the average sum rate (over terminal locations and channel realizations) with instantaneous CSI and no spatial correlation is shown as a function of the SNR. In the case of $N_{t}=4$, the DVSINR approach is superior to MRT and ZF at most SNRs, although ZF approaches DVSINR at very high cell edge SNR. The performance loss compared with optimal precoding is at most $15-20 \%$, depending on the SNR, and will asymptotically approach zero since DVSINR achieves the optimal multiplexing gain (see Theorem 5). This raises the question of whether the high backhaul demands for achieving the optimal solution are justifiable in practice. In the case of $N_{t}=2$ (with the power allocation in [20, eq. (10)]), the performance of both DVSINR and MRT saturates at high SNR since 


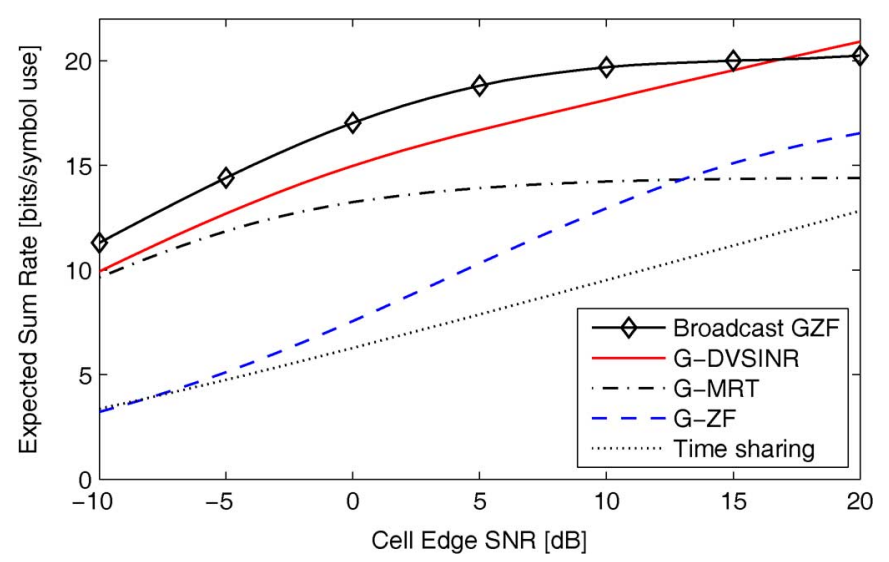

Fig. 5. Expected sum rate (over terminal locations and channel statistics) in a system with local statistical CSI, $K_{t}=K_{r}=4, N_{t}=6$, and an angular spread of 10 degrees (as seen from each base station). The scenario considers uniformly distributed terminals within a square with base stations in each corner.

$K_{r}>N_{t}$, but DVSINR still constitutes a major performance improvement compared with MRT. Distributed ZF does not exist for this number of antennas.

In Fig. 5, the expected sum rate (over terminal locations) with $N_{t}=6$, statistical CSI, and an angular spread of 10 degrees (as seen from a transmitter) is shown as a function of the SNR. The G-DVSINR approach in Strategy 3, G-MRT, and G-ZF (all using the power allocation in Strategy 4 with $g_{j k}^{\text {symmetric }}$ ) are compared with equal time sharing between the terminals and an upper bound consisting of the broadcast GZF approach in [28] that requires both statistical CSI and perfect instantaneous norm feedback. In this scenario, the G-DVSINR approach is clearly the better choice among the distributed methods; it even beats the upper bound at high SINR, since the performance GZF approach saturates at around $15 \mathrm{~dB}$ SNR. All the cooperative approaches outperform time sharing.

\section{CONCLUSION}

We have considered cooperative multicell precoding in a system with an arbitrary number of multi-antenna transmitters and single-antenna receivers. The outer boundary of the achievable rate region was characterized for transmitters with either instantaneous or statistical CSI. At each transmitter, the spans of beamforming vectors that can attain this boundary only depend on local CSI, and can be interpreted as linear combinations of MRT and ZF vectors. This enables distributed precoding in a structured manner that only requires local CSI and processing. By viewing the multicell system as a superposition of broadcast channels, we propose a novel framework of distributed virtual SINR (DVSINR) beamforming that satisfies the optimal beamforming characterization and achieves the optimal multiplexing gain. It was applied for distributed beamforming with instantaneous and statistical CSI, along with two heuristic power allocation schemes. The performance of this approach was illustrated and shown to combine the benefits of conventional MRT and ZF, and outperform them at most SNRs. Finally, the loss in performance of having only local CSI is rather small, compared with the backhaul and computational demands of sharing and processing global CSI.

\section{APPENDIX A \\ COLLECTION OF LEMMAS AND PROOFS}

Lemma 3: Let $b_{k} \in \mathcal{C N}\left(0, \mu_{k}\right)$ be independent random variables with distinct variances $\mu_{k}>0$ for $k=1, \ldots, K$, and let $\sigma_{k}^{2} \geq 0$. Then

$$
\begin{aligned}
& \mathbb{E}\left\{\log _{2}\left(\sigma_{k}^{2}+\sum_{k=1}^{K}\left|b_{k}\right|^{2}\right)\right\} \\
& =\log _{2}\left(\sigma_{k}^{2}\right)+\sum_{k=1}^{K} \frac{e^{\frac{\sigma_{k}^{2}}{\mu_{k}}} E_{1}\left(\frac{\sigma_{k}^{2}}{\mu_{k}}\right)}{\log (2) \prod_{l \neq k}\left(1-\frac{\mu_{l}}{\mu_{k}}\right)}
\end{aligned}
$$

where $E_{1}(x)=\int_{1}^{\infty} e^{-x u} / u d u$ is the exponential integral.

Proof: Let $z=\sum_{k=1}^{K}\left|b_{k}\right|^{2} / \sigma_{k}^{2}$ and observe that

$$
\begin{aligned}
& \mathbb{E}\left\{\log _{2}\left(\sigma_{k}^{2}+\sum_{k=1}^{K}\left|b_{k}\right|^{2}\right)\right\} \\
& =\log _{2}\left(\sigma_{k}^{2}\right)+\int_{0}^{\infty} \frac{\log (1+z)}{\log (2)} \sum_{k=1}^{K} \frac{\sigma_{k}^{2} e^{-\frac{\sigma_{k}^{2}}{\mu_{k}}}}{\mu_{k} \prod_{l \neq k}\left(1-\frac{\mu_{l}}{\mu_{k}}\right)} d z
\end{aligned}
$$

using the PDF expression for $z$ in [23, (5)]. The integrand that contains $z$ is

$$
\begin{aligned}
\int_{0}^{\infty} & \log (1+z) e^{-\frac{\sigma_{k}^{2}}{\mu_{k}} z} d z \\
= & \int_{1}^{\infty} \log (\tilde{z}) e^{-\frac{\sigma_{k}^{2}}{\mu_{k}}(\tilde{z}-1)} d \tilde{z} \\
= & e^{\frac{\sigma_{k}^{2}}{\mu_{k}}}(\underbrace{\left.\left[-\frac{\mu_{k}}{\sigma_{k}^{2}} \log (\tilde{z}) e^{-\frac{\sigma_{k}^{2}}{\mu_{k}} \tilde{z}}\right]_{1}^{\infty}+\frac{\mu_{k}}{\sigma_{k}^{2}} E_{1}\left(\frac{\sigma_{k}^{2}}{\mu_{k}}\right)\right)}_{=0}
\end{aligned}
$$

where the first equality follows from the variable substitution $\tilde{z}=1+z$ and the second from integration by parts. Substitution into (26) gives the final expression.

Proof of Theorem 1: Using the notation for the SINR in (4), the expected rate can be divided as

$$
\begin{aligned}
\mathbb{E} & \left\{\log _{2}\left(1+\operatorname{SINR}_{k}\right)\right\} \\
= & \mathbb{E}\left\{\log _{2}\left(\sum_{\bar{k}=1}^{K_{r}}\left|a_{\bar{k} k}\right|^{2}+\sigma_{k}^{2}\right)\right\} \\
- & \mathbb{E}\left\{\log _{2}\left(\sum_{\substack{k=1 \\
k \neq k}}^{K_{r}}\left|a_{\bar{k} k}\right|^{2}+\sigma_{k}^{2}\right)\right\} .
\end{aligned}
$$

Observe that $\left\|\mathbf{a}_{k}\right\|^{2}=\sum_{\bar{k}=1}^{K_{r}}\left|a_{\bar{k} k}\right|^{2}$ and since the Euclidean norm is invariant under unitary transformations, $\sum_{\bar{k}=1}^{\operatorname{rank}\left(\mathbf{S}_{k}\right)}\left|b_{\bar{k}}\right|^{2}$ has identical distribution for independent variables $b_{\bar{k}} \in \mathcal{C N}\left(0, \mu_{\bar{k}}\right)$. Using these variables, we can apply Lemma 3 and achieve the first term in (5). The second term 
is achieved by a similar transformation based on eigenvalues of $\tilde{\mathbf{S}}_{k}$.

Proof of Theorem 2: Consider a rate tuple $\left(R_{1}, \ldots, R_{K_{r}}\right)$ on the Pareto boundary that is achieved by beamforming vectors $\mathbf{w}_{j k}$ and power allocation $p_{j k}$ for all $j, k$. The following approach can be taken (for each $j, k$ ) to replace $\mathbf{w}_{j k}$ with a beamformer that fulfills (8) and reduces the power usage, while achieving the same rate tuple. Let $\mathcal{A}_{j k}=\left\{\mathbf{h}_{j k}\right\} \bigcup_{\bar{k} \neq k}\left\{\boldsymbol{\Pi}_{\mathbf{h}_{j k}}^{\perp} \mathbf{h}_{j k}\right\}$ and observe that the vector $\mathbf{w}_{j k}$ can be expressed as the linear combination

$$
\mathbf{w}_{j k}=\gamma_{k} \mathbf{h}_{j k}+\sum_{\substack{k=1 \\ k \neq k}}^{K_{r}} \gamma_{\bar{k}} \boldsymbol{\Pi}_{\mathbf{h}_{j k}}^{\perp} \mathbf{h}_{j k}+\sum_{l=\operatorname{rank}\left(\mathcal{A}_{j k}\right)+1}^{N_{t}} \gamma_{l} \mathbf{v}_{l}
$$

for some complex-valued coefficients $\gamma_{k}$ and some orthogonal basis $\left\{\mathbf{v}_{l}\right\}_{l=\operatorname{rank}\left(\mathcal{A}_{j k}\right)+1}^{N_{t}}$ for the orthogonal complement to $\mathcal{A}_{j k}$. Now, observe that

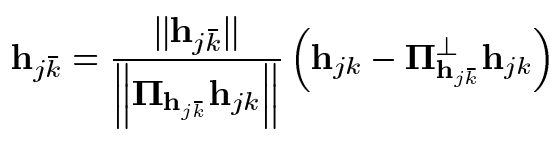

for all $\bar{k}$ that are nonorthogonal to $\mathbf{h}_{j k}$ (while orthogonal channels can be removed from $\mathcal{A}_{j k}$, since these directions only create interference). Thus, $\mathbf{h}_{j \bar{k}}^{H} \mathbf{v}_{l}=0$ for $\bar{k}=1, \ldots, K_{r}$ and $l=$ $\operatorname{rank}\left(\mathcal{A}_{j k}\right)+1, \ldots, N_{t}$. Since $\mathbf{w}_{j k}$ only appears in the SINR expression in (3) as inner products with $\mathbf{h}_{j k}$ and $\mathbf{h}_{j \bar{k}}$, the identical rate tuple is achieved by the beamforming vector

$$
\begin{aligned}
\tilde{\mathbf{w}}_{j k}=\frac{\gamma_{k}}{\sqrt{1-\sum_{l=\operatorname{rank}\left(\mathcal{A}_{j k}\right)+1}^{N_{t}}\left|\gamma_{l}\right|^{2}}} \mathbf{h}_{j k} \\
\quad+\sum_{\substack{k=1 \\
k \neq k}}^{K_{r}} \frac{\gamma_{\bar{k}}}{\sqrt{1-\sum_{l=\operatorname{rank}\left(\mathcal{A}_{j k}\right)+1}^{N_{t}}\left|\gamma_{l}\right|^{2}}} \mathbf{\Pi}_{\mathbf{h}_{j k}}^{\perp} \mathbf{h}_{j k}
\end{aligned}
$$

and transmit power $\tilde{p}_{j k}=p_{j k}\left(1-\sum_{l=\operatorname{rank}\left(\mathcal{A}_{j k}\right)+1}^{N_{t}}\left|\gamma_{l}\right|^{2}\right) \leq$ $p_{j k}$. Thus, we have proved that all rate tuples on the Pareto boundary can be achieved by beamforming vectors $\tilde{\mathbf{w}}_{j k} \in \operatorname{span}\left(\mathcal{A}_{j k}\right)$.

Next, we will show that if $\mathbf{h}_{j k} \notin \operatorname{span}\left(\bigcup_{\bar{k} \neq k}\left\{\mathbf{h}_{j \bar{k}}\right\}\right)$ for some $j, k$, then $\mathrm{BS}_{j}$ needs to use full power to reach the Pareto boundary. The given property corresponds to that $\sum_{l=1}^{m_{j k}} \boldsymbol{\Pi}_{\mathbf{e}_{j k}^{(l)}} \mathbf{h}_{j k} \neq \mathbf{h}_{j k}$, where $\mathbf{e}_{j k}^{(1)}, \ldots, \mathbf{e}_{j k}^{\left(m_{j k}\right)}$ is an orthogonal basis of $\operatorname{span}\left(\bigcup_{\bar{k} \neq k}\left\{\mathbf{h}_{j \bar{k}}\right\}\right)$. Consequently, there should exist a zero-forcing vector $\mathrm{u}=\left(\mathbf{I}-\sum_{l=1}^{m_{j k}} \boldsymbol{\Pi}_{\mathbf{e}_{j k}^{(l)}}\right) \mathbf{h}_{j k} \neq \mathbf{0}$ that satisfies $\mathbf{h}_{j \bar{k}}^{H} \mathrm{u}=0$ for all $\bar{k} \neq k$.

Now, assume for the purpose of contradiction that the Pareto boundary is attained for a set of beamforming vectors $\left\{\mathbf{w}_{j k}\right\}$ and power allocations $\left\{p_{j k}\right\}$ that fulfills $\sum_{k=1}^{K_{r}} p_{j k}<P_{j}$. Then, we can replace $\mathbf{w}_{j k}$ and $p_{j k}$ by

$$
\begin{aligned}
p_{j k}^{\text {new }} & =P_{j}-\sum_{\substack{k=1 \\
k \neq k}}^{K_{r}} p_{j \bar{k}} \quad \text { and } \\
\mathbf{w}_{j k}^{\text {new }} & =\sqrt{\frac{p_{j k}}{p_{j k}^{\text {new }}}} \mathbf{w}_{j k}+\alpha u e^{i \arg \left(\mathbf{h}_{j k}^{H} \mathbf{w}_{j k}\right)}
\end{aligned}
$$

for some positive parameter $\alpha$ that makes $\left\|\mathbf{w}_{j k}^{\text {new }}\right\|=1$. This corresponds to increasing the power in the zero-forcing direction and making sure that the signal powers add up constructively at the intended receiver. Thus, $\mathrm{BS}_{j}$ can increase the signal power at $\mathrm{MS}_{k}$ as $p_{j k}^{\text {new }}\left|\mathbf{h}_{j k}^{H} \mathbf{w}_{j k}^{\text {new }}\right|^{2}=$ $p_{j k}\left(\left|\mathbf{h}_{j k}^{H} \mathbf{w}_{j k}\right|+\alpha \mathbf{h}_{j k}^{H} \mathrm{u}\right)^{2}>p_{j k}\left|\mathbf{h}_{j k}^{H} \mathbf{w}_{j k}\right|^{2}$, without affecting the co-terminal interference. In other words, $R_{k}$ has been increased without affecting $R_{\bar{k}}$ for $\bar{k} \neq k$, which is a contradiction to the assumption that the initial rate tuple belonged to the Pareto boundary. Thus, full transmit power is required to attain the Pareto boundary. The condition in (8) also becomes a necessary condition, because otherwise we can decrease the power by the approach in the first part of the proof and then increased it again using (31).

Proof of Theorem 3: Consider an expected rate tuple $\left(\mathbb{E}\left\{R_{1}\right\}, \ldots, \mathbb{E}\left\{R_{K_{r}}\right\}\right)$ on the Pareto boundary that is achieved by beamforming vectors $\mathbf{w}_{j k}$ and power allocation $p_{j k}$ for all $j, k$. The beamformers $\mathbf{w}_{j k}$ can in general be expressed as the linear combination

$$
\mathbf{w}_{j k}=\sum_{l=1}^{m} \gamma_{l} \mathbf{v}_{l}+\sum_{l=m+1}^{N_{t}} \gamma_{l} \mathbf{u}_{l}
$$

where $\left\{\mathbf{v}_{l}\right\}_{l=1}^{m}$ is an orthogonal basis of the $m$-dimensional given by $\operatorname{span}\left(\bigcup_{k=1}^{K_{r}}\left\{\mathbf{U}_{j k}^{(D)}\right\}\right)$ and $\left\{\mathrm{u}_{l}\right\}_{l=m+1}^{N_{t}}$ is an orthogonal basis of the orthogonal complement. The coefficients $\gamma_{l}$ are complex-valued and fulfill $\sum_{l=1}^{N_{t}}\left|\gamma_{l}\right|^{2}=1$, since $\mathbf{w}_{j k}$ is expanded in terms of an orthonormal basis. To avoid allocating power to the weak eigenvalues in the orthogonal complement, we can replace $\mathbf{w}_{j k}$ by

$$
\tilde{\mathbf{w}}_{j k}=\frac{1}{\sqrt{\sum_{l=1}^{m}\left|\gamma_{l}\right|^{2}}} \sum_{l=1}^{m} \gamma_{l} \mathbf{v}_{l}
$$

and reduce the transmit power to $\tilde{p}_{j k}=p_{j k} \sum_{l=1}^{m}\left|\gamma_{l}\right|^{2} \leq p_{j k}$. This new precoding satisfy (11) and will achieve a new rate tuple $\left(\mathbb{E}\left\{\tilde{R}_{1}\right\}, \ldots, \mathbb{E}\left\{\tilde{R}_{K_{r}}\right\}\right)$. Next, we show that the difference in performance is bounded by $o\left(\epsilon_{k}\right)$. With the new precoding, the change in the covariance matrix $\mathbf{S}_{k}$ in (4) is limited since $\mathbf{S}_{k}=\sum_{j=1}^{K_{t}} \tilde{\mathbf{W}}_{j}^{H} \mathbf{Q}_{j k} \tilde{\mathbf{W}}_{j}+\mathbf{E}$, where $\tilde{\mathbf{W}}_{j}=\left[\sqrt{\tilde{p}_{j 1}} \tilde{\mathbf{w}}_{j 1} \cdots \sqrt{\tilde{p}_{j K_{r}}} \tilde{\mathbf{w}}_{j K_{r}}\right]$ and the elements of the symmetric perturbation matrix $\mathbf{E}$ are bounded as $o\left(\epsilon_{k}\right)$. By applying the eigenvalue perturbation result in ([31], Theorem 7.2.2) when deriving $\mathbb{E}\left\{\tilde{R}_{k}\right\}$ in (5), the eigenvalues $\mu_{m}$ and $\lambda_{m}$ can be replaced by $\tilde{\mu}_{m}=\mu_{m}+o\left(\epsilon_{k}\right)$ and $\tilde{\lambda}_{m}=\lambda_{m}+o\left(\epsilon_{k}\right)$, respectively. Observe that each term in (5) has the structure

$$
\begin{gathered}
\frac{e^{\frac{\sigma_{k}^{2}}{\mu_{m}+o\left(\epsilon_{k}\right)}} E_{1}\left(\frac{\sigma_{k}^{2}}{\mu_{m}+o\left(\epsilon_{k}\right)}\right)}{\log (2) \prod_{l \neq m}\left(1-\frac{\mu_{l}+o\left(\epsilon_{k}\right)}{\mu_{m}+o\left(\epsilon_{k}\right)}\right)} \\
=\frac{e^{\frac{\sigma_{k}^{2}}{\mu_{m}}} E_{1}\left(\frac{\sigma_{k}^{2}}{\mu_{m}}\right)}{\log (2) \prod_{l \neq m}\left(1-\frac{\mu_{l}}{\mu_{m}}\right)}+o\left(\epsilon_{k}\right)
\end{gathered}
$$

where the equality follows from straightforward appliance of l'Hospital's rule. Thus, by applying this result to each 
term in (5), we achieve $\mathbb{E}\left\{R_{k}\right\}=\mathbb{E}\left\{\tilde{R}_{k}\right\}+o\left(\epsilon_{k}\right)$. To finalize the proof of the first part, observe that for arbitrary covariance matrices it holds with probability one that $\operatorname{span}\left(\boldsymbol{\Pi}_{\mathbf{U}_{j \bar{k}}^{(D)}} \mathbf{U}_{j k}^{(D)}\right)=\operatorname{span}\left(\mathbf{U}_{j \bar{k}}^{(D)}\right)$ for all $\bar{k}$. Since,

$$
\boldsymbol{\Pi}_{\mathbf{U}_{j \bar{k}}^{(D)}} \mathbf{U}_{j k}^{(D)}=\left(\mathbf{I}-\boldsymbol{\Pi}_{\mathbf{U}_{j \bar{k}}^{(D)}}^{\perp}\right) \mathbf{U}_{j k}^{(D)}
$$

it follows that

$\operatorname{span}\left(\bigcup_{k=1}^{K_{r}}\left\{\mathbf{U}_{j k}^{(D)}\right\}\right)=\operatorname{span}\left(\left\{\mathbf{U}_{j k}^{(D)}\right\} \bigcup_{\bar{k} \neq k}\left\{\Pi_{\mathbf{U}_{j \bar{k}}^{(D)}}^{\perp} \mathbf{U}_{j k}^{(D)}\right\}\right)$.

Finally, consider the case when $\operatorname{span}\left(\bigcup_{k=1}^{K_{r}}\left\{\mathbf{U}_{j k}^{(D)}\right\}\right) \neq \mathbb{C}^{N_{t}}$ for some $j$. If $\sum_{k} p_{j k}<P_{j}$, we propose the following way of increasing the power usage while guaranteeing the same type performance. We assume that the beamforming vector $\mathbf{w}_{j k}$ fulfills (11), otherwise we can follow the approach in first part of the proof to decrease the power usage, retain the performance, and fulfill (11). Select a unit vector $\mathbf{d} \notin \operatorname{span}\left(\bigcup_{k=1}^{K_{r}}\left\{\mathbf{U}_{j k}^{(D)}\right\}\right)$. If we replace the beamformer and the power allocation with

$$
\begin{aligned}
p_{j k}^{\text {new }} & =P_{j}-\sum_{\bar{k}=1, \bar{k} \neq k}^{K_{r}} p_{j \bar{k}} \text { and } \\
\mathbf{w}_{j k}^{\text {new }} & =\sqrt{\frac{p_{j k}}{p_{j k}^{\text {new }}}} \mathbf{w}_{j k}+\sqrt{1-\frac{p_{j k}}{p_{j k}^{\text {new }}}} \mathbf{d}
\end{aligned}
$$

the difference in signal and interference variance yields a perturbation in $\mathbf{S}_{k}$ on the order of $o\left(\epsilon_{k}\right)$ and we can use the approach above to show that resulting rate tuple fulfills $\mathbb{E}\left\{R_{k}\right\}=$ $\mathbb{E}\left\{\tilde{R}_{k}\right\}+o\left(\epsilon_{k}\right)$. Hence, full transmit power can be used to achieve $\left(\mathbb{E}\left\{\tilde{R}_{1}\right\}, \ldots, \mathbb{E}\left\{\tilde{R}_{K_{r}}\right\}\right)$.

Proof of Theorem 4: Let $R_{k}^{\text {opt }}=\log _{2}\left(1+\operatorname{SINR}_{k}^{\text {opt }}\right)$ represent an arbitrary Pareto optimal rate tuple. Observe that this rate tuple is achieved by solving

$$
\begin{aligned}
\underset{\mathbf{w}_{j k} \in \mathbb{C}^{N_{t}}, p_{j k} \geq 0 \forall j, k}{\operatorname{maximize}} & \min _{1 \leq k \leq K_{r}} \frac{R_{k}}{R_{k}^{\mathrm{opt}}} \\
\text { subject to } & \sum_{k=1}^{K_{r}} p_{j k} \leq P_{j},\left\|\mathbf{w}_{j k}\right\|^{2}=1 \text { for all } k
\end{aligned}
$$

since [19, Lemma 1] shows that all solutions to (36) must satisfy $R_{1} / R_{1}^{\mathrm{opt}}=\ldots=R_{K_{r}} / R_{K_{r}}^{\mathrm{opt}}$. Thus, with $R_{1}^{\mathrm{opt}}, \ldots, R_{K_{r}}^{\mathrm{opt}}$ as rate constraints for the different terminals, the uplink-downlink duality result of ([19, , Th. 1]) can be applied. This means that the optimal beamforming vectors for the downlink problem in (36) should also maximize the virtual uplink SINRs in (14) for each user, and the parameters $\beta_{j k}$ represents the optimal power allocation in the virtual dual uplink.

Proof of Theorem 5: Let the arbitrary power allocation be denoted $p_{j k}=P_{j} \tilde{p}_{j k}$ with (normalized) coefficients $\tilde{p}_{j k}$. As $P=\min _{j} P_{j}$, we let $P_{j}=P \alpha_{j}$ for some parameters $0<\alpha_{j}<$
1 for all $j$. Using the SINR expression in (3) and the given power allocation, the sum rate becomes

$$
\begin{aligned}
& \sum_{k=1}^{K_{r}} \log _{2}\left(1+\frac{\left|\sum_{j=1}^{K_{t}} \sqrt{P \alpha_{j} \tilde{p}_{j k}} \mathbf{h}_{j k}^{H} \mathbf{w}_{j k}^{(\text {DVSINR })}\right|^{2}}{\sum_{\substack{k=1 \\
k \neq k}}^{K_{r}}\left|\sum_{j=1}^{K_{t}} \sqrt{P \alpha_{j} \tilde{p}_{j \bar{k}}} \mathbf{h}_{j k}^{H} \mathbf{w}_{j \bar{k}}^{(\text {DVSINR })}\right|^{2}+\sigma_{k}^{2}}\right) \\
& =K_{r} \log _{2}(P) \\
& +\sum_{k=1}^{K_{r}} \log _{2}\left(\frac{1}{P}+\frac{\left|\sum_{j=1}^{K_{t}} \sqrt{\alpha_{j} \tilde{p}_{j k}} \mathbf{h}_{j k}^{H} \mathbf{w}_{j k}^{(\text {DVSINR })}\right|^{2}}{\sum_{\substack{k=1 \\
k \neq k}}^{K_{r}}\left|\sum_{j=1}^{K_{t}} \sqrt{P \alpha_{j} \tilde{p}_{j \bar{k}}} \mathbf{h}_{j k}^{H} \mathbf{w}_{j \bar{k}}^{(\text {DVSINR })}\right|^{2}+\sigma_{k}^{2}}\right) .
\end{aligned}
$$

If $N_{t} \geq K_{r}$, then with probability $\mathbf{h}_{j k} \notin \operatorname{span}\left(\bigcup_{\bar{k} \neq k}\left\{\mathbf{h}_{j \bar{k}}\right\}\right)$ for all $j, k$. By analyzing the expression $\mathbf{w}_{j \bar{k}}^{\text {(DVSINR) }}=$ $\mathbf{C}_{j \bar{k}}^{-1} \mathbf{h}_{j \bar{k}} /\left\|\mathbf{C}_{j \bar{k}}^{-1} \mathbf{h}_{j \bar{k}}\right\|$ it is straightforward to show that $\sqrt{P} \mathbf{h}_{j k}^{H} \mathbf{w}_{j \bar{k}}^{(\mathrm{DVSINR})} \rightarrow 0$ as $P \rightarrow \infty$. Thus, the last term of (37) is bounded as $P \rightarrow \infty$ and the multiplexing gain is $K_{r}$.

\section{ACKNOWLEDGMENT}

The authors would like to thank the reviewers for their insightful comments and suggestions, which have strengthened the analysis and improved the precoding performance.

\section{REFERENCES}

[1] D. Gesbert, M. Kountouris, R. Heath, C.-B. Chae, and T. Sälzer, "Shifting the MIMO paradigm," IEEE Signal Process. Mag., vol. 24, no. 5, pp. 36-46, 2007.

[2] S. Shamai and B. Zaidel, "Enhancing the cellular downlink capacity via co-processing at the transmitting end," in Proc. IEEE VTC'01-Spring, 2001, vol. 3, pp. 1745-1749.

[3] H. Zhang and H. Dai, "Cochannel interference mitigation and cooperative processing in downlink multicell multiuser MIMO networks,' EURASIP J. Wireless Commun. Netw., vol. 2, pp. 222-235, 2004.

[4] M. Karakayali, G. Foschini, and R. Valenzuela, "Network coordination for spectrally efficient communications in cellular systems," IEEE Wireless Commun. Mag., vol. 13, no. 4, pp. 56-61, 2006.

[5] S. Venkatesan, A. Lozano, and R. Valenzuela, "Network MIMO: Overcoming intercell interference in indoor wireless systems," in Proc. IEEE ACSSC'07, 2007, pp. 83-87.

[6] P. Viswanath and D. Tse, "Sum capacity of the vector Gaussian broadcast channel and uplink-downlink duality," IEEE Trans. Inf. Theory, vol. 49, no. 8, pp. 1912-1921, 2003.

[7] W. Yu, "Uplink-downlink duality via minimax duality," IEEE Trans. Inf. Theory, vol. 52, no. 2, pp. 361-374, 2006.

[8] P. Marsch and G. Fettweis, "On multicell cooperative transmission in backhaul-constrained cellular systems," Ann. Telecommun., vol. 63, pp. 253-269, 2008

[9] S. Jing, D. Tse, J. Soriaga, J. Hou, J. Smee, and R. Padovani, "Multicell downlink capacity with coordinated processing," EURASIP J. Wireless Commun. Netw., 2008.

[10] B. L. Ng, J. Evans, S. Hanly, and D. Aktas, "Distributed downlink beamforming with cooperative base stations," IEEE Trans. Inf. Theory, vol. 54, no. 12, pp. 5491-5499, 2008.

[11] O. Simeone, O. Somekh, H. V. Poor, and S. Shamai, "Downlink multicell processing with limited-backhaul capacity," EURASIP J. Adv. Signal Process., 2009. 
[12] M. Kobayashi, M. Debbah, and J. Belfiore, "Outage efficient strategies in network MIMO with partial CSIT," in Proc. IEEE ISIT'09, 2009, pp. 249-253.

[13] E. Larsson and E. Jorswieck, "Competition versus cooperation on the MISO interference channel," IEEE J. Sel. Areas Commun., vol. 26, no. 7, 2008.

[14] E. Jorswieck, E. Larsson, and D. Danev, "Complete characterization of the Pareto boundary for the MISO interference channel," IEEE Trans. Signal Process., vol. 56, no. 10, 2008.

[15] N. Hassanpour, J. Smee, J. Hou, and J. Soriaga, "Distributed beamforming based on signal-to-caused-interference ratio," in Proc. IEEE ISSSTA'08, 2008, pp. 405-410.

[16] B. O. Lee, H. W. Je, I. Sohn, O.-S. Shin, and K. B. Lee, "Interferenceaware decentralized precoding for multicell MIMO TDD systems," in Proc. IEEE GLOBECOM'08, 2008.

[17] R. Zakhour and D. Gesbert, "Coordination on the MISO interference channel using the virtual SINR framework," in Proc. Int. ITG Workshop Smart Antennas, 2009.

[18] E. Björnson, R. Zakhour, D. Gesbert, and B. Ottersten, "Distributed multicell and multiantenna precoding: Characterization and performance evaluation," in Proc. IEEE GLOBECOM'09, 2009.

[19] M. Schubert and H. Boche, "Solution of the multiuser downlink beamforming problem with individual SINR constraints," IEEE Trans. Veh. Technol., vol. 53, no. 1, pp. 18-28, 2004.

[20] E. Björnson and B. Ottersten, "On the principles of multicell precoding with centralized and distributed cooperation," in Proc. WCSP'09, 2009.

[21] R. Zakhour and D. Gesbert, "Distributed multicell-MISO precoding using the layered virtual SINR framework," IEEE Trans. Wireless Commun., accepted for publication.

[22] E. Björnson and B. Ottersten, "A framework for training-based estimation in arbitrarily correlated Rician MIMO channels with Rician disturbance," IEEE Trans. Signal Process., vol. 58, no. 3, pp. 1807-1820, 2010.

[23] E. Björnson, D. Hammarwall, and B. Ottersten, "Exploiting quantized channel norm feedback through conditional statistics in arbitrarily correlated MIMO systems," IEEE Trans. Signal Process., vol. 57, no. 10, pp. 4027-4041, 2009.

[24] E. Björnson and B. Ottersten, "Post-user-selection quantization and estimation of correlated Frobenius and spectral channel norms," in Proc. IEEE PIMRC'08, 2008.

[25] J. Lindblom, E. Karipidis, and E. Larsson, "Selfishness and altruism on the MISO interference channel: The case of partial transmitter CSI," IEEE Commun. Lett., vol. 13, no. 9, pp. 667-669, 2009.

[26] D. Chizhik, J. Ling, P. Wolniansky, R. Valenzuela, N. Costa, and K. Huber, "Multiple-input-multiple-output measurements and modeling in Manhattan," IEEE J. Sel. Areas Commun., vol. 21, no. 3, pp. 321-331, 2003.

[27] K. Yu, M. Bengtsson, B. Ottersten, D. McNamara, P. Karlsson, and M. Beach, "Modeling of wide-band MIMO radio channels based on NLoS indoor measurements," IEEE Trans. Veh. Technol., vol. 53, no. 3, pp. $655-665,2004$

[28] D. Hammarwall, M. Bengtsson, and B. Ottersten, "Utilizing the spatial information provided by channel norm feedback in SDMA systems," IEEE Trans. Signal Process., vol. 56, no. 7, pp. 3278-3293, 2008.

[29] H. Zhang, N. Mehta, A. Molisch, J. Zhang, and H. Dai, "Asynchronous interference mitigation in cooperative base station systems," IEEE Trans. Wireless Commun., vol. 7, no. 1, pp. 155-165, 2008.

[30] S. Boyd and L. Vandenberghe, Convex Optimization. Cambridge, U.K.: Cambridge Univ. Press, 2004.

[31] G. Golub and C. V. Loan, Matrix Computations. Baltimore, MD: The Johns Hopkins Univ. Press, 1996.

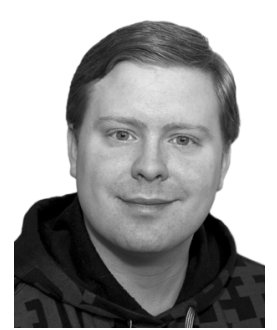

Emil Björnson (S'07) was born in Malmö, Sweden, in 1983 . He received the M.S. degree in engineering mathematics from Lund University, Lund, Sweden, in 2007. He is currently working towards the $\mathrm{Ph} . \mathrm{D}$. degree in telecommunications at the Signal Processing Laboratory, KTH Royal Institute of Technology, Stockholm, Sweden.

His research interests include wireless communications, resource allocation, estimation theory, stochastic signal processing, and mathematical optimization.

Mr. Björnson received a Best Paper Award at the 2009 International Conference on Wireless Communications and Signal Processing (WCSP 2009) for his work on multicell MIMO communications.

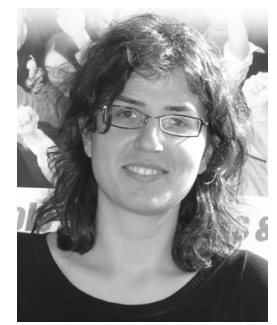

Randa Zakhour (S'00) received the B.E. degree in computer and communications engineering from the American University of Beirut, Lebanon, in 2002. In June 2006, she received the M.Sc. degree in communications engineering from the RWTH Aachen, Germany. She received the Ph.D. degree in electronics and communications from Telecom ParisTech in April 2010, where she carried out her doctoral research work at the Mobile Communications Department at EURECOM, Sophia Antipolis.

Her current research interests include signal processing, optimization, and feedback and resource allocation issues in multicell multiuser MIMO systems.

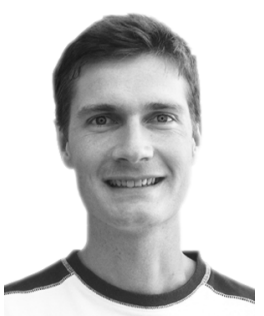

David Gesbert (S'96-M'99-SM'05) received the Ph.D. degree from Ecole Nationale Superieure des Telecommunications, France, in 1997.

From 1997 to 1999 he has been with the Information Systems Laboratory, Stanford University. In 1999, he was a founding engineer of Iospan Wireless Inc, San Jose, Ca., a startup company pioneering MIMO-OFDM (now Intel). Between 2001 and 2003 he has been with the Department of Informatics, University of Oslo as an adjunct professor. He is currently a Professor in the Mobile Communications Department, EURECOM, France, where he heads the Communications Theory Group. He has published about 170 papers and several patents all in the area of signal processing, communications, and wireless networks.

Dr. Gesbert was a Co-Editor of several special issues on wireless networks and communications theory, for the IEEE JOURNAL OF SELECTED AREAS IN COMMUNICATIONS (2003, 2007, and 2010), EURASIP Journal on Applied Signal Processing (2004 and 2007), Wireless Communications Magazine (2006). He served on the IEEE Signal Processing for Communications Technical Committee, 2003-2008. He is an Associate Editor for IEEE TRANSACTIONS ON WIRELESS COMMUNICATIONS and the EURASIP Journal on Wireless Communications and Networking. He authored or coauthored papers winning the 2004 IEEE Best Tutorial Paper Award (Communications Society) for a 2003 JSAC paper on MIMO systems, 2005 Best Paper (Young author) Award for Signal Proc. Society journals, and the Best Paper Award for the 2004 ACM MSWiM workshop. He coauthored the book Space Time Wireless Communications: From Parameter Estimation to MIMO Systems (Cambridge University Press, 2006).

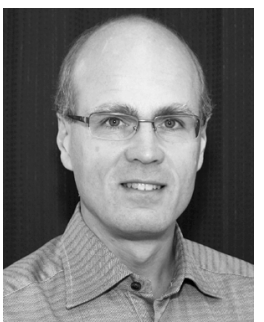

Björn Ottersten (S'87-M'89-SM'99-F'04) was born in Stockholm, Sweden, in 1961. He received the M.S. degree in electrical engineering and applied physics from Linköping University, Linköping, Sweden, in 1986 and the Ph.D. degree in electrical engineering from Stanford University, Stanford, CA, in 1989.

He has held research positions at the Department of Electrical Engineering, Linköping University; the Information Systems Laboratory, Stanford University; and the Katholieke Universiteit Leuven, Leuven, Belgium. During 1996-1997, he was Director of Research at ArrayComm Inc., San Jose, CA, a start-up company based on Otterstens patented technology. In 1991 , he was appointed Professor of Signal Processing at the Royal Institute of Technology (KTH), Stockholm, Sweden. From 2004 to 2008, he was Dean of the School of Electrical Engineering at KTH, and from 1992 to 2004, he was head of the Department for Signals, Sensors, and Systems at KTH. He is also Director of security and trust at the University of Luxembourg. His research interests include wireless communications, stochastic signal processing, sensor array processing, and time-series analysis.

Dr. Ottersten has coauthored papers that received an IEEE Signal Processing Society Best Paper Award in 1993, 2001, and 2006. He has served as Associate Editor for the IEEE TRANSACTIONS ON SigNAL PROCESSING and on the Editorial Board of the IEEE Signal Processing Magazine. He is currently Editor-in-Chief of the EURASIP Signal Processing Journal and a member of the Editorial Board of the EURASIP Journal of Advances in Signal Processing. $\mathrm{He}$ is a Fellow of EURASIP. He is one of the first recipients of the European Research Council advanced research grant. 